\title{
A comprehensive analysis using 9 dark matter halo models on the spiral galaxy NGC 4321
}

\author{
Tan Wei Shen ${ }^{1 *}$. Zamri Zainal Abidin ${ }^{1}$. \\ Norsiah Hashim ${ }^{2}$.
}

Received: date / Accepted: date

\begin{abstract}
This paper addressed the dark matter analysis on the spiral galaxy NGC 4321 (M100) by considering the nine different dark matter profiles, so far lacking in the scientific literature, i.e. Pseudoisothermal, Burkert, NFW, Moore, Einasto, core-modified, DC14, coreNFW and Lucky13 profiles. In this paper, we analyzed the rotation curve analysis on the galaxy NGC 4321 by using nonlinear fitting of star, gaseous and dark matter halo equations with selected VLA HI observation data. Among the nine dark matter profiles, four dark matter profiles (DC14, Lucky13, Burkert and Moore profiles) showed declining features and hence not suitable for this galaxy. This is concluded to be mainly due to the characteristics of those dark matter profiles and also the varying levels of problems within the inner region fittings. For the remaining five accepted dark matter profiles, we further conducted the analysis by using reduced chi-square test. Four out of the five accepted dark matter profiles lie within the range of $0.40<\chi_{\text {red }}^{2}<1.70$, except for the core-modified profile. In addition, Pseudoisothermal profile achieved the best fitting with $\chi_{r e d}^{2}$ nearest to 1 , mainly due to its linearity in the inner region and flatness at large radii.
\end{abstract}

Keywords Cosmology · Dark matter · Radioastronomy · Spiral galaxy

PACS 98.80.-k · 95.35.+d · 95.85.Bh, 95.85.Fm $\cdot 98.52 . \mathrm{Nr}, 98.56 . \mathrm{Ne}$

\author{
Tan Wei Shen \\ ${ }^{1}$ Radio Cosmology Research Lab, Physics Dept., Faculty of Science, University of Malaya, \\ Kuala Lumpur, Malaysia \\ E-mail: weishen06@hotmail.com \\ Zamri Zainal Abidin \\ ${ }^{1}$ Radio Cosmology Research Lab, Physics Dept., Faculty of Science, University of Malaya, \\ Kuala Lumpur, Malaysia \\ E-mail: zzaa@um.edu.my \\ Norsiah Hashim \\ ${ }^{2}$ Mathematics Section, Centre for Foundation Studies in Science, University of Malaya, \\ Kuala Lumpur, Malaysia \\ E-mail: norsiahashim@um.edu.my
}




\section{Introduction}

Dark matter is one of the most important investigations since there are still a lot of mysteries related to it. In galaxies, the distribution of the baryonic components cannot justify the observed profiles or the amplitudes of the measured circular velocities [1]. Furthermore, the profiles of the rotation curve imply that the distribution of light does not match the distribution of mass within the galaxies [2]. The explanation of the lacking distribution leads to the suggestions of a presence of an additional invisible mass component [3]. This is usually solved by adding an extra mass component, i.e. the dark matter halo [4].

Though initial evidence for dark matter came from rotation curves of galaxies, more compelling evidence for non-baryonic matter exists. In 1980, Vera Rubin and Kent Ford present the observations of a set of spiral galaxies that orbital velocities of stars in galaxies were unexpectedly high at large distances from the nucleus by using the new sophisticated optical spectrograph that they developed [5]. This unexpected result indicated that the falloff in luminous mass with distance from the centre is balanced by an increase in nonluminous mass. Although initially met with skepticism, Rubin's result of the existence of dark matter became scientifically accepted after the subsequent decades by studying more than 200 galaxies and enough documented data proved that the universe was virtually 90 percent undiscovered matter [3].

NGC 4321 (also known as M100) is a grand design galaxy located in the Virgo cluster. NGC 4321 is an SAB(s)bc galaxy that has two symmetric welldefined spiral arms [6]. M100 has receive much observational and theoretical attention, not only because it is one of the closest galaxies in Virgo cluster, but also because its bar of moderate strength gives rise to a particularly clear resonant circumnuclear structure [7]. Its relative proximity and moderate inclination make it suitable to study the content, distribution and kinematics of the neutral hydrogen gas in both its molecular (CO) and atomic (HI) forms of its interstellar medium [8].

Rotation of spiral galaxies is measured by spectroscopic observations of emission lines such as $\mathrm{H} \alpha, \mathrm{HI}$ and $\mathrm{CO}$ lines. In these lines, the velocity dispersion is negligibly small compared to rotational velocity. This implies that the pressure term in the Virial theorem is also negligible so that the mass can be calculated in sufficient accuracy by the dynamical balance between the gravitational and centrifugal forces [9]. HI rotation curves are most often derived from velocity fields. A velocity field aims to give a compact and accurate shorthand description of the dynamics of a galaxy by assigning a typical velocity to every spatial position. That is, for every position, one uses the velocity that most accurately represents the circular motion of the bulk of the quiescent component of the gas as it moves around the center of the galaxy [10]. Fitting the inner part of the rotation curve is challenging. For the case of M100, the existence of intermediate bar [11] and the known starburst activity in the center of this galaxy [12] may induced non circular motions. 
A large number of $\mathrm{HI}$ observation studies in the broader literature have examined this galaxy, namely, HI rotation curves for this galaxy were derived by Vera Rubin $[1,3]$, HI observation by VLA and Nobeyama radio observatory to study large-scale star-formation processes [13], HI rotation curves to see the behavior of the approaching and receding sides of NGC 4321 by Knapen [14] and study of environmental effects on HI gas properties of cluster galaxies [15]. Besides the usage of HI observational data to measure the galaxy's rotation curves, $\mathrm{CO}$ and $\mathrm{H} \alpha$ have also been previously used for this purpose since 1980, namely, central CO rotation curves of this galaxy derived by Sofue [16], $\mathrm{H} \alpha$ rotation curves and Position-Velocity diagram by Daigle [17] and $\mathrm{H} \alpha$ rotation curves of the inner disc by Morales [18] and CO rotation curve to study the dark matter in the central region by using ALMA [19].

Previous CO rotation curve studies by Ali [19] have exclusively focused on the dark matter in the central region of this galaxy, which is defined to be up to the radius $0.7 \mathrm{kpc}$ with NFW dark matter profile. The unexpected findings of the dark matter in the central region signal the need for additional studies to understand more deeply about the overall dark matter distribution in this galaxy. To fill this literature gap, this paper will employ HI observed data up to radius $10 \mathrm{kpc}$ to identify the overall dark matter distribution by using a rotation curve with the nine different dark matter profiles. The nine dark matter profiles that we used are Pseudoisothermal, Burkert, NFW, Moore, Einasto, core-modified, DC14, coreNFW and Lucky13 dark matter profiles. More detailed information about the nine dark matter profiles will be discussed in Methodology section.

\section{Methodology}

This section is organized as follows: In subsection 2.1 we introduce the mass modeling of rotation curve technique and then each parameter in the subsequent subsection. The HI observed data processing is explained in subsection 2.2. In subsection 2.3 , we present the observed data calculation by using the tilted-ring method. The rotation curve comparison with previous works is explained in subsection 2.4. In the last few subsections, we discuss the mass model parameter of the nine dark matter profiles, stellar, and gas in subsection $2.5,2.6$, and 2.7 , respectively.

\subsection{Mass modeling rotation curve}

The measurement of the rotation curves of disc galaxies is a powerful tool to investigate the nature of dark matter [20]. Rotation curve has been used to assess the existence, the amount and the distribution of this dark component [4]. Most galaxies have rotation curves that show sharp rising or high velocity in the very centre, following by a slowly rising or constant velocity rotation in the outer parts. The flatness of the rotation curves in the outer part implies 


\begin{tabular}{|l|l|}
\hline Parameter & Value \\
\hline \hline Observation date & 25 th March 2003 \\
\hline Total observation time & 12910 seconds \\
\hline Total antennas used & 27 \\
\hline Antennas diameter (each) & $25.0 \mathrm{~m}$ \\
\hline Total data recorded & 855738 \\
\hline Polarizations & LL and RR \\
\hline Configurations & $\mathrm{D}$ \\
\hline Band & $\mathrm{L}$ \\
\hline RA & $12: 22: 54$ \\
\hline Dec & $+15: 49: 20$ \\
\hline Systemic velocity & $1575 \mathrm{kms}$ \\
\hline Position Angle & $-26^{\circ}$ \\
\hline Rest Frequency & $1420.41 \mathrm{MHz}$ \\
\hline Total bandwidth observation & $1538.1 \mathrm{kHz}$ \\
\hline Divided channel & 63 \\
\hline Each channel frequency & $24.41 \mathrm{kHz}$ \\
\hline Restoring beam (major, minor) & $\left(52.95^{\prime \prime}, 47.66^{\prime \prime}\right)$ \\
\hline Velocity resolution & $10 \mathrm{kms}^{-1}$ \\
\hline Primary calibrator & $1331+305$ \\
\hline Secondary calibrator & $1221+282$ \\
\hline
\end{tabular}

Table 1: HI observational parameter of VLA on galaxy NGC 4321

that galaxies contain large amounts of dark matter [21]. The contribution of each component to the rotation curve is computed by using Mathematica software. By summing in quadrature the contribution of the three components (disc, gas, dark matter) in all possible combinations and get the best-fitting to the observed data, we will obtain the most accurate value of star, gas and dark matter [22]. The rotation curve can be represented as a model, which is the sum of the contribution from the star, halo and gas components [23] as follows:

$$
V_{\text {rot }}^{2}=V_{\text {gas }}^{2}+V_{\text {star }}^{2}+V_{D M}^{2}
$$

\subsection{VLA (Very Large Array) HI data}

The observed data is NGC $4321 \mathrm{HI}$ (neutral atomic hydrogen) $21 \mathrm{~cm}$ line from VLA archive data with project number AS0750_D030325. The data reduction for inspection, flagging, bandpass calibration, gain calibration, continuum subtraction, cleaning, imaging, moment mapping and PV (PositionVelocity) Diagram generating is processed by using CASA (Common Astronomy Software Application) software. The distance adopted is 17.1 Mpc [24]. The overall HI observational parameters of VLA on galaxy NGC 4321 are detailed in Table 1.

Figure 1 shows the velocity channel map of $\mathrm{HI}$ in the central region of NGC 4321 with restoring beam of 52.95 " $\times 47.66$ " was produced by using the Briggs weighting. The HI emission is detected in 28 channel maps with a velocity width of $10 \mathrm{kms}^{-1}$ from $1436 \mathrm{kms}^{-1}$ to $1706 \mathrm{kms}^{-1}$. The pixel size is set to CELL $=15 "$ and IMSIZE $=256$. Then, the channel map is used as input to generate the integrated intensity $\mathrm{HI}$ zeroth moment (mom0) map as shown in 
Figure 2. The outcome of Figure 1 and Figure 2 is comparable with the previous study (refer to [25] Figure 2 and Figure 3). Next, the integrated-intensity HI map is used to generate PV diagram and obtain the HI velocity of the galaxy as shown in Figure 3.

\subsection{Data calculation by using tilted-ring method}

Next, the tilted-ring method is used in order to obtain the rotation velocity. By using the tilted-ring method, the rotation velocity, radial velocity and inclination angle in a galactic disk are coupled to each other [26] as below:

$$
V_{r}(r, \theta)=V_{\text {obs }}(r, \theta)-V_{\text {sys }}=V_{\text {rot }}(r) \cos \theta \sin i
$$

where $V_{r}(r, \theta)$ is the radial velocity due to rotational motion, $V_{o b s}(r, \theta)$ is the observed radial velocity, $r$ is the radius from the center of the galaxy, $\theta$ is the azimuth angle in the disk of a measured point from the major axis, $V_{\text {sys }}$ is the systemic velocity of the galaxy, $V_{\text {rot }}(r)$ is the rotation velocity and $i$ is the inclination angle of the galaxy.

Any coupling of rotation velocity and the inclination can be solved by using the tilted-ring method if a velocity field is observed [1,27-29]. This is because of the functional shape of the variation of $V_{r}(r, \theta) / V_{r}(r, 0)$ is against the position angle on the sky. In this situation, $V_{r}(r, 0)$ is the maximum value of $V_{r}$ along an initially chosen ring [26]. For $\theta=0, V_{\text {rot }}(r)=V_{r}(r, 0) / \cos 0 \sin$ $i$, where $V_{r}(r, 0)=V_{\text {obs }}(r, 0)-V_{\text {sys }}$. The systemic velocity and inclination angle of M100 is $1575 \mathrm{kms}^{-1}$ [7] and $27^{\circ}$ [14] respectively. The complete rotation velocity calculation of M100 is shown in Table 2.

In Table 2, column 1 illustrates the radius, $r$ of M100, column 2 reveals the observed velocity, $V_{\text {obs }}$ of M100, obtained from Figure 3 VLA PV diagram. Column 3 demonstrates the radial velocity, $V_{r}$ of M100 by using observed velocity, $V_{\text {obs }}$ minus systemic velocity, $V_{\text {sys }}\left(1575 \mathrm{kms}^{-1}\right)$. While column 4 shows the rotation velocity, $V_{\text {rot }}$ of M100 by using radial velocity, $V_{r}$ divides $\sin i\left(27^{\circ}\right)$. Then, a graph of the total rotation velocity against the radius of M100 is drawn as shown in Figure 4. In Figure 4, the error bars of the observed data are obtained from the rms values of the VLA data, which the root mean square is often use as a synonym for standard deviation of a signal from a given baseline.

\subsection{Rotation curve comparison}

Our rotation curve is then compared with Rubin et al. [3] and Knapen et al. [14] rotation curves. Rubin and Knapen only showed rotation velocity and did not decompose it into separate contribution of gas, star and dark matter. Hence, we were only able to compare the total rotation velocities between Rubin, Knapen and our rotation curves.

For Rubin rotation curve, the values of the rotation velocities are available in their paper, we have included their rotation velocities in the Table 3. For 

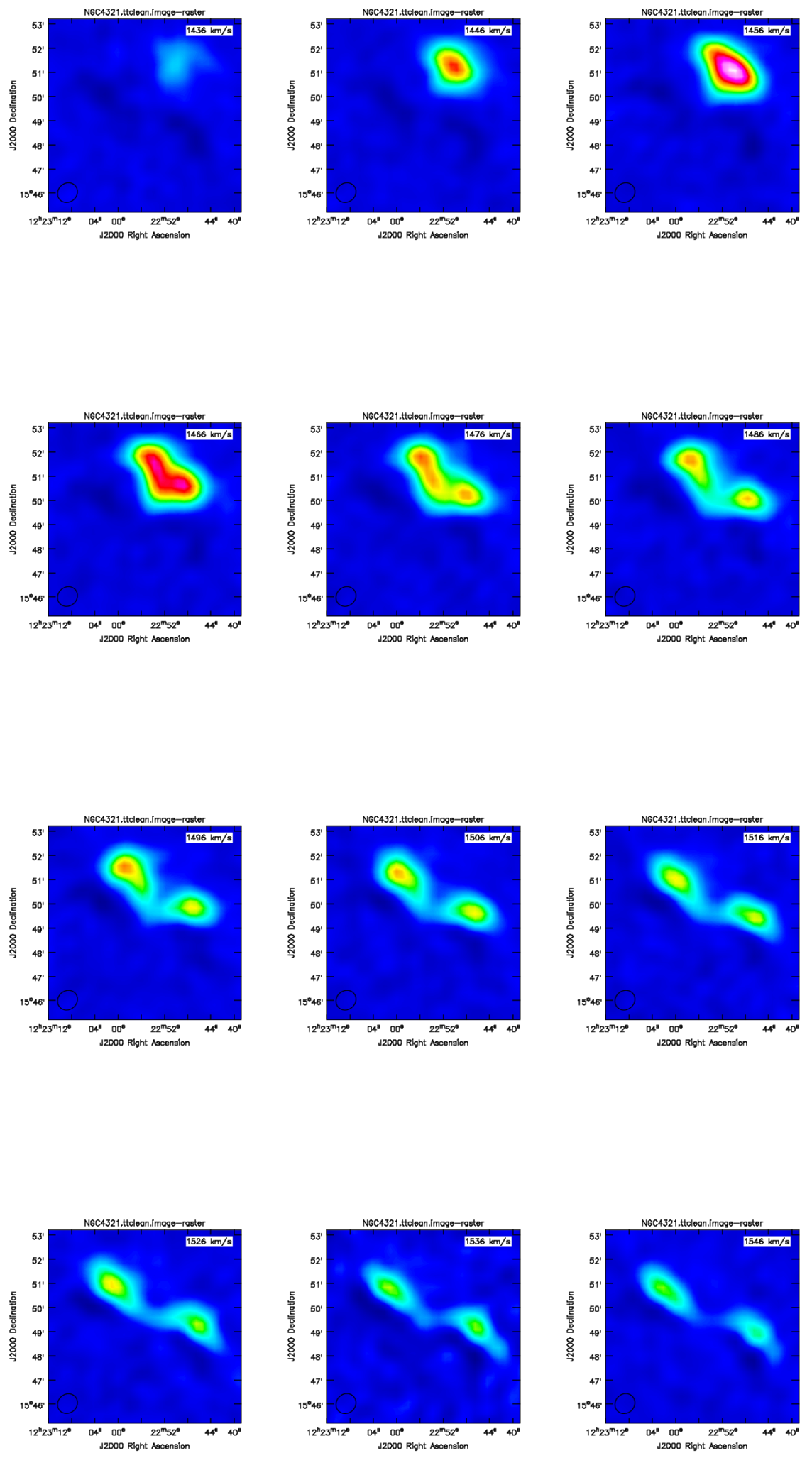

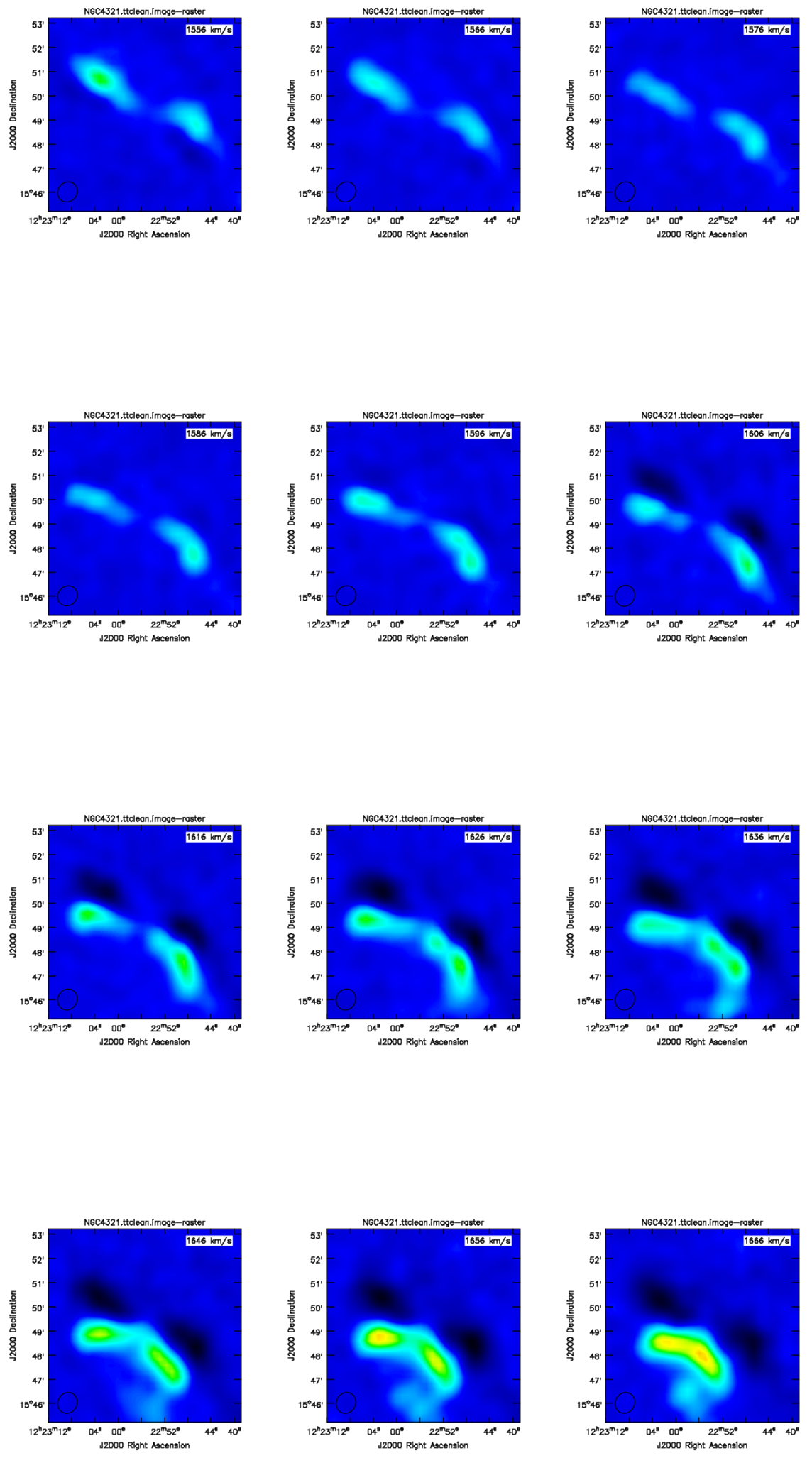

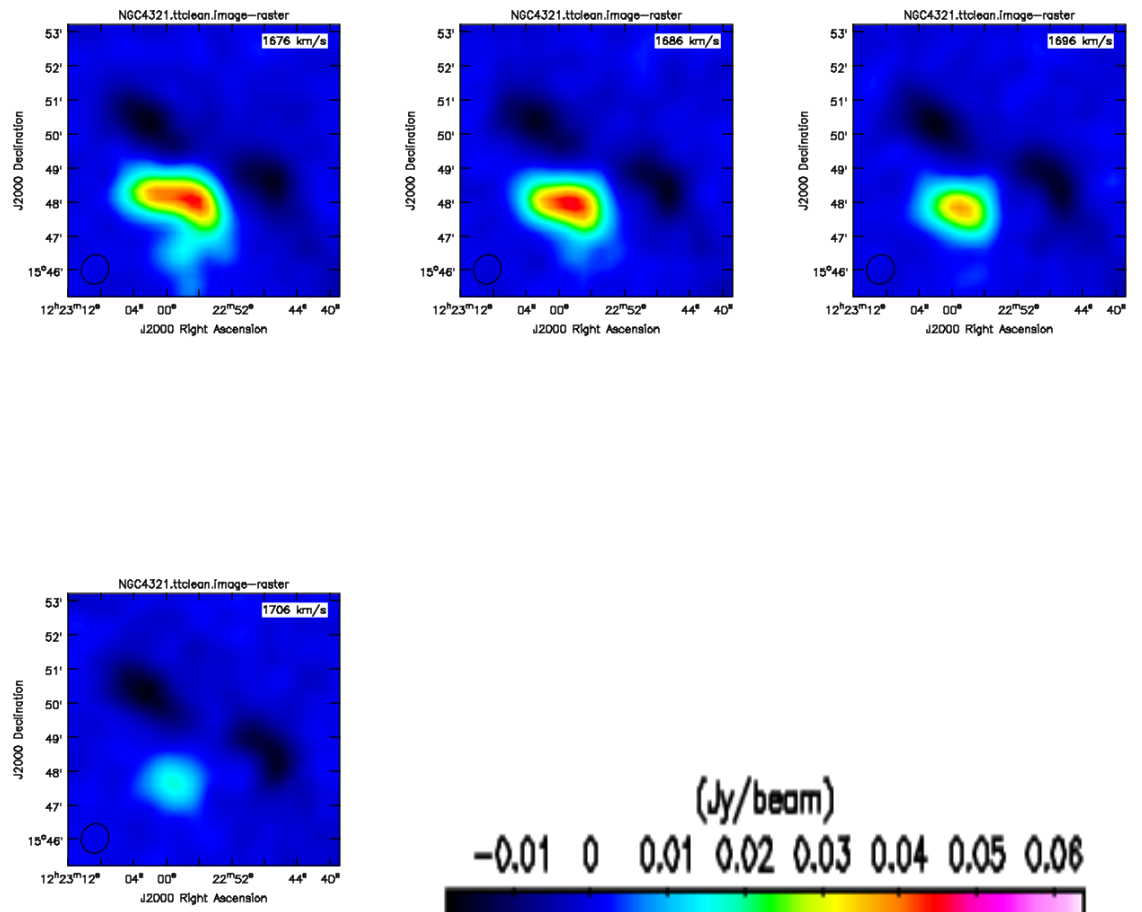

\section{$(\mathrm{Jy} / \mathrm{beam})$}

$\begin{array}{llllllllll}-0.01 & 0 & 0.01 & 0.02 & 0.03 & 0.04 & 0.05 & 0.06\end{array}$

Fig. 1 Velocity channel map of HI in the central region of NGC 4321

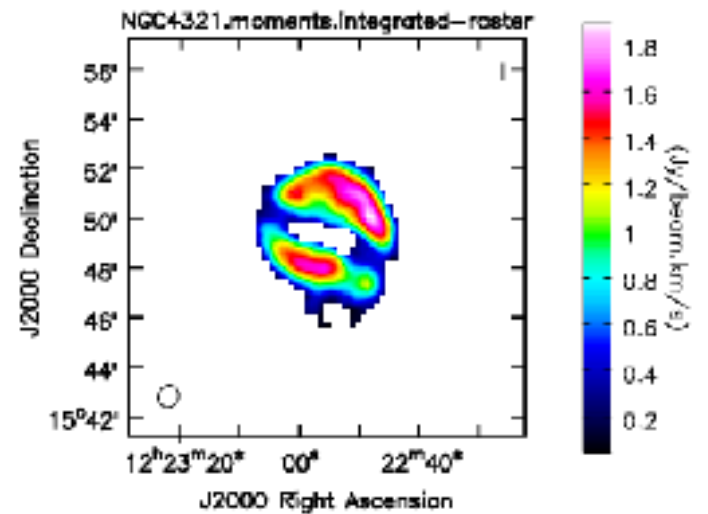

Fig. 2 Integrated intensity (mom0) map of the HI $21 \mathrm{~cm}$ line emission of NGC 4321 


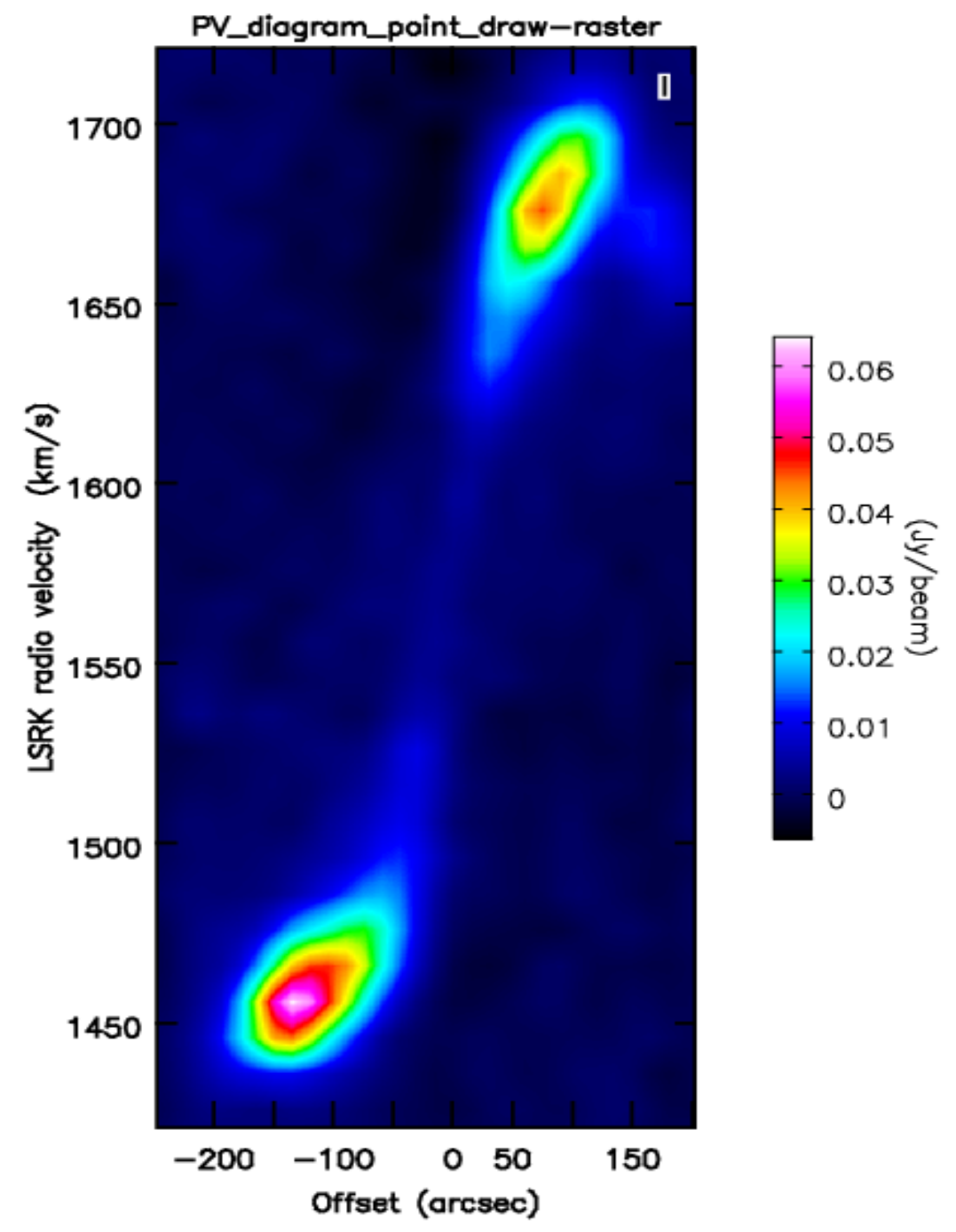

Fig. 3 NGC 4321 PV diagram

Knapen rotation curve, they did not include the values of rotation velocities from their paper, so we will trace the rotation velocity value from the figure in their paper. We standardized all the relevant units from the two said papers and our manuscript. The total rotation velocity against the radius of Rubin, Knapen and our rotation curve is hence shown in Table 3. The three rotation curves are plotted together in Figure 5. 


\begin{tabular}{|c|c|c|c|}
\hline Radius, $\mathrm{r}(\mathrm{kpc})$ & $V_{\text {obs }}\left(\mathrm{kms}^{-1}\right)$ & $V_{\text {obs }}-V_{\text {sys }}\left(\mathrm{kms}^{-1}\right)$ & $\begin{array}{l}V_{\text {rot }}=\left(V_{\text {obs }}-V_{\text {sys }}\right) / \text { sin } \\
\left(\mathrm{kms}^{-1}\right)\end{array}$ \\
\hline 0.02 & 1586.42 & 11.42 & 25.15 \\
\hline 0.41 & 1603.5 & 28.50 & 62.78 \\
\hline 0.83 & 1614.27 & 39.27 & 86.50 \\
\hline 1.24 & 1622.81 & 47.81 & 105.31 \\
\hline 1.66 & 1631.35 & 56.35 & 124.12 \\
\hline 2.07 & 1637.49 & 62.49 & 137.65 \\
\hline 2.49 & 1643.8 & 68.80 & 151.55 \\
\hline 2.90 & 1649 & 74.00 & 163.00 \\
\hline 3.31 & 1652.71 & 77.71 & 171.17 \\
\hline 3.73 & 1656.42 & 81.42 & 179.34 \\
\hline 4.14 & 1660.5 & 85.50 & 188.33 \\
\hline 4.56 & 1664.96 & 89.96 & 198.15 \\
\hline 4.97 & 1668.3 & 93.30 & 205.51 \\
\hline 5.39 & 1671.27 & 96.27 & 212.05 \\
\hline 5.80 & 1674.24 & 99.24 & 218.59 \\
\hline 6.21 & 1675.73 & 100.73 & 221.88 \\
\hline 6.63 & 1678.33 & 103.33 & 227.60 \\
\hline 7.04 & 1679.81 & 104.81 & 230.86 \\
\hline 7.46 & 1681.66 & 106.66 & 234.94 \\
\hline 7.87 & 1684.71 & 109.71 & 241.66 \\
\hline 8.29 & 1686.55 & 111.55 & 245.71 \\
\hline 8.70 & 1688.38 & 113.38 & 249.74 \\
\hline 9.11 & 1690.21 & 115.21 & 253.77 \\
\hline 9.53 & 1691.44 & 116.44 & 256.48 \\
\hline 9.94 & 1693.27 & 118.27 & 260.51 \\
\hline 2.14 & & & \\
\hline
\end{tabular}

Table 2: The radius, observed velocity, radial velocity and rotation velocity of galaxy M100

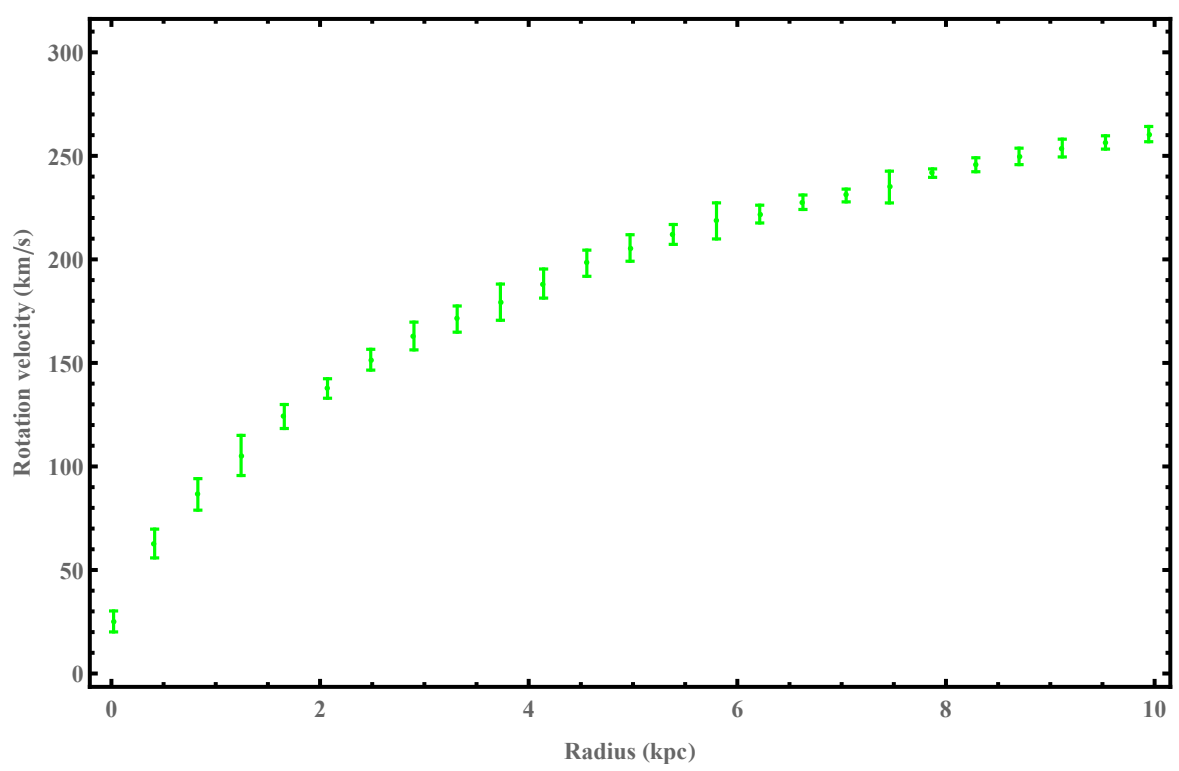

Fig. 4 The total rotation velocity against the radius of M100 


\begin{tabular}{|c|c|c|c|}
\hline Radius (kpc) & \multicolumn{3}{|c|}{ Total rotation velocity $\left(\mathrm{kms}^{-1}\right)$} \\
\hline & Rubin et al. 1980 [3] & Knapen et al. 1993 [14] & Our rotation curve \\
\hline 0 & 0 & 0 & 0 \\
\hline 1 & 133 & 159 & 96 \\
\hline 2 & 124 & 205 & 137 \\
\hline 3 & 158 & 211 & 168 \\
\hline 4 & 182 & 216 & 185 \\
\hline 5 & 188 & 229 & 206 \\
\hline 6 & 190 & 231 & 220 \\
\hline 7 & 193 & 233 & 230 \\
\hline 8 & 197 & 247 & 243 \\
\hline 9 & 199 & 259 & 252 \\
\hline 10 & 201 & 265 & 262 \\
\hline
\end{tabular}

Table 3: The comparison of the rotation velocity obtained by Rubin et al., Knapen et al. and our rotation curve

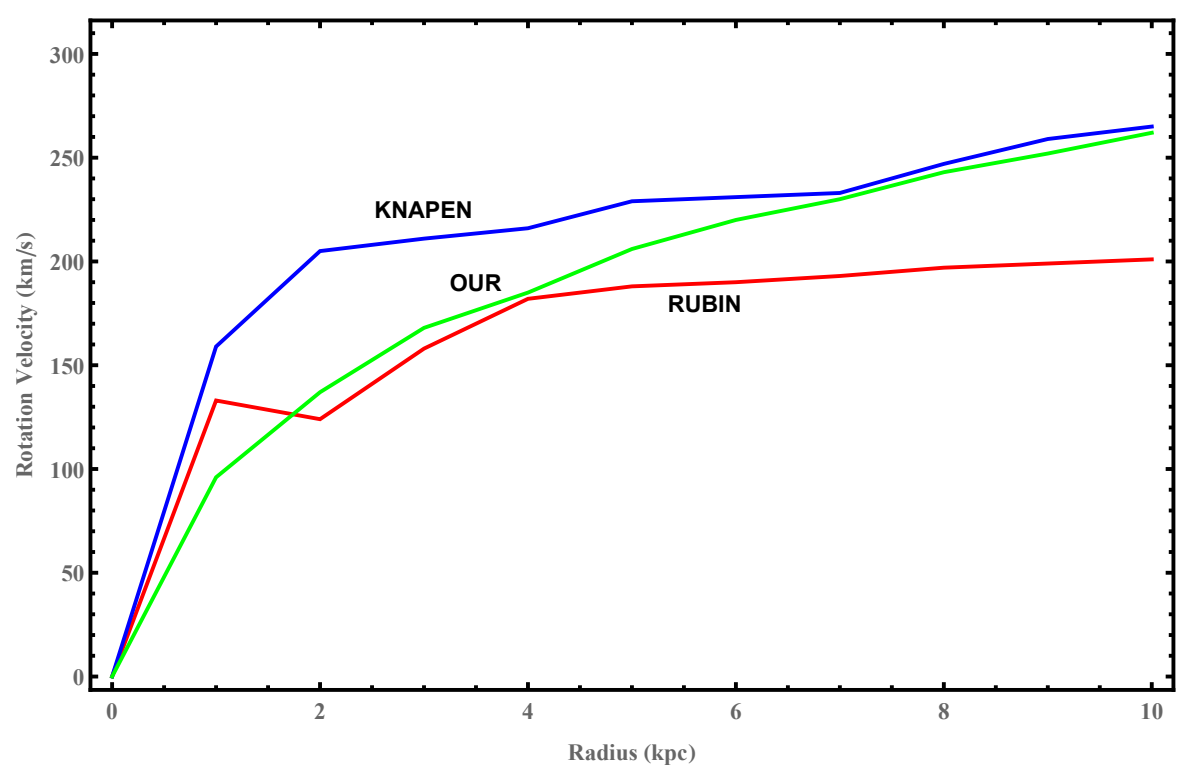

Fig. $\quad 5$ The total rotation velocity comparison between Rubin, Knapen and our rotation curve. The red line represents Rubin rotation curve, the blue line represents Knapen rotation curve and the green line represents our rotation curve.

By referring to Figure 5, the reasons of Rubin rotation curve to be different from Knapen and our rotation curves are due to the differences of systemic velocity, position angle and inclination angle that were applied. The comparison is shown in Table 4 .

The rotation curve of Vera Rubin was observed in the HII regions emission from the Kitt Peak $4 \mathrm{~m}$ RC spectrograph in 1978 [3], while the rotation curve of Knapen and ours were observed in the $21 \mathrm{~cm}$ line of neutral hydrogen with Very Large Array (VLA) in 1990 and 2003, respectively. In Table 4, the difference in position angle will affect the Position-Velocity diagram obtained 


\begin{tabular}{|l|l|l|l|}
\hline Parameter & Rubin et al. 1980 [3] & Knapen et al. 1993 [14] & This paper \\
\hline $\begin{array}{l}\text { Systemic Velocity } \\
\left(\mathrm{kms}^{-1}\right)\end{array}$ & $1545 \pm 25$ & $1570.8 \pm 0.8$ & 1575 \\
\hline Position angle $\left(^{\circ}\right)$ & 140 & $153 \pm 1$ & $\begin{array}{l}-26 \\
\text { (same as 154) }\end{array}$ \\
\hline $\begin{array}{l}\text { Inclination } \\
\text { angle }\left(^{\circ}\right)\end{array}$ & 35 & 27 & 27 \\
\hline
\end{tabular}

Table 4: The comparison of parameter adopted between Rubin et al, Knapen et al. and in this paper

during data reduction. It is worth noting that the differences in systemic velocity and inclination angle will affect the calculation for rotation velocity. This is because the tilted ring method formulation involves both systemic velocity and inclination angle. The uncertainty of Rubin is far larger than the uncertainty of Knapen. The reason is that different instrument is used and normally instruments improved in their uncertainty calculations over the years.

Furthermore, the measurement methods vary between Knapen and ours. They produced maps with a resolution of 12.7 " $\times 13.3$ " uniform weighting and $31.75 " \times 28.75$ " natural weighting by using AIPS software [14]. However, we produced images with a resolution of 52.95 " $\times 47.66$ " Briggs weighting by using CASA software. Natural weighting is applied to all visibilities equally and results in maximum point-source sensitivity in images. However, this weighting produces poor synthesized beam-shape and side-lobe levels. The uniform weighting gives the visibilities a weight that is inversely proportional to the sampling density function. This causes a reduction in the side-lobes of the PSF. The uniform weighting scheme provides better resolution but lowers the sensitivity of the image [30]. Both natural and uniform weightings have some weak points but the use of Briggs weighting presents a way to overcome these weaknesses. Briggs weighting creates a PSF that smoothly varies between natural and uniform weightings based on the signal-to-noise ratio of the measurements and a tunable parameter that defines a noise threshold [31], which produced better image. Furthermore, for Knapen rotation curve central region, they did mention that their curve rises more steeply near the center. This is because their profile was measured by a high full width at half maximum within a beam of half-power width, which corresponds to a rapidly rising rotation curve [14]. Beyond $2 \mathrm{kpc}$ radius (0.4 arcmin), the rapidly rising rotation velocity has slowed down and, eventually, starting from $6 \mathrm{kpc}$ radius, the rotation velocity of Knapen rotation curve is comparable with the rotation velocity of our rotation curve.

\subsection{Dark Matter Profile}

Researchers expand the dark matter study with a larger theoretical framework to provide better ways of directly investigating it, since then a lot of dark matter profiles have been derived. In our research of rotation curve-fitting on the galaxy NGC 4321, we will consider the nine dark matter profiles including 
seven cored and two cuspy profiles to analyze the distribution and mass of dark matter halo. Cored profiles that we used are Pseudoisothermal, Burkert, Einasto, core-modified, DC14, coreNFW and Lucky13 profiles. While for cuspy profiles, we used Navarro, Frenk and White (NFW) and Moore profiles.

\subsubsection{Pseudoisothermal profile}

Pseudoisothermal profile is a singular density profile that approaches a power law at the centre [32] and the mass distribution for larger radii would diverge proportional to the radius. For Pseudoisothermal profile, the density, mass and velocity equations of dark matter [33] are as follows:

$$
\begin{gathered}
\rho_{\text {Iso }}(r)=\frac{\rho_{0}}{1+\left(r / r_{s}\right)^{2}} \\
M_{I s o}(r)=4 \pi \rho_{0} r_{s}^{2}\left(r-r_{s} \tan ^{-1}\left(\frac{r}{r_{s}}\right)\right) \\
V_{I s o}^{2}(r)=4 \pi G \rho_{0} r_{s}^{2}\left(1-\frac{r_{s}}{r} \tan ^{-1}\left(\frac{r}{r_{s}}\right)\right)
\end{gathered}
$$

where parameter $G$ is the universal gravitational constant, $\rho_{0}$ is the scale density, $r_{s}$ is the scale radius and $r$ is the radius from the centre of the galaxy. These parameter definitions are the same for all others profiles except Einasto and DC14 profiles. Hence, these parameter definitions will not be mentioned again in the following profiles.

\subsubsection{Burkert profile}

Burkert profile revises density law of Pseudoisothermal profile in the inner region. This represents the mass profile for larger radii that diverge logarithmically with increasing radius. This is in agreement with the predictions of cosmological CDM calculations [34]. For Burkert profile, the density [34], mass [35] and velocity equations [36] of dark matter are as follows:

$$
\begin{gathered}
\rho_{\text {Bur }}(r)=\frac{\rho_{0} r_{s}{ }^{3}}{\left(r+r_{s}\right)\left(r^{2}+r_{s}^{2}\right)} \\
\left.M_{B u r}(r)=6.4 \rho_{0} r_{s}^{3}\left[\ln \left(1+\frac{r}{r_{s}}\right)-\tan ^{-1}\left(\frac{r}{r_{s}}\right)\right)+0.5 \ln \left(1+\left(\frac{r}{r_{s}}\right)^{2}\right)\right] \\
V_{\text {Bur }}^{2}(r)=\frac{6.4 G \rho_{0} r_{s}^{3}}{r}\left(\ln \left[\left(1+\frac{r}{r_{s}}\right)\left(1+\left(\frac{r}{r_{s}}\right)^{2}\right)^{0.5}\right]-\tan ^{-1}\left(\frac{r}{r_{s}}\right)\right)
\end{gathered}
$$




\subsubsection{Navarro, Frenk and White (NFW) profile}

NFW profile is a traditional benchmark profile motivated by N-body simulations, which is shallower than Pseudoisothermal near the centre, and steeper than Pseudoisothermal in the outer regions [37]. For NFW profile, the density [37], mass [38] and velocity equation [36] of dark matter are as follows:

$$
\begin{gathered}
\rho_{N F W}(r)=\rho_{0} \frac{r_{s}}{r}\left(1+\frac{r}{r_{s}}\right)^{-2} \\
M_{N F W}(r)=4 \pi \rho_{0} r_{s}^{3}\left(\ln \left(1+\frac{r}{r_{s}}\right)-\frac{r}{r_{s}+r}\right) \\
V_{N F W}^{2}(r)=\frac{12.6 G \rho_{0} r_{s}^{3}}{r}\left(\ln \left(1+\frac{r}{r_{s}}\right)-\frac{r}{r_{s}+r}\right)
\end{gathered}
$$

\subsubsection{Moore profile}

Moore profile behaves similarly to the NFW profile at large radii but is steeper than NFW profile at smaller radii [39]. For Moore profile, the density [40], mass [41] and velocity equations of dark matter are as follows:

$$
\begin{gathered}
\rho_{M o o}(r)=\frac{\rho_{0}}{\left(\frac{r}{r_{s}}\right)^{1.5}\left(1+\left(\frac{r}{r_{s}}\right)^{1.5}\right)} \\
M_{M o o}(r)=\frac{8}{3} \pi \rho_{0} r_{s}^{3}\left(\ln \left(1+\left(\frac{r}{r_{s}}\right)^{1.5}\right)\right) \\
V_{M o o}^{2}(r)=\frac{8.38 G \rho_{0} r_{s}^{3}}{r}\left(\ln \left(1+\left(\frac{r}{r_{s}}\right)^{1.5}\right)\right)
\end{gathered}
$$

\subsubsection{Einasto profile}

Einasto profile is emerging as a better fit for more recent numerical simulations and provides the most accurate description of dark matter haloes [42]. Compared to other dark matter profiles use two free parameters, Einasto profile uses three free parameters to describe the halo mass profile instead and hence significantly improves the accuracy of the fitting to the inner density profiles of simulated haloes [43]. For Einasto profile, the density, mass [44] and velocity equations of dark matter are as follows:

$$
\begin{gathered}
\rho_{\text {Ein }}(r)=\rho_{-2} \exp \left[-2 n\left(\left(\frac{r}{r_{-2}}\right)^{1 / n}-1\right)\right] \\
M_{E i n}(r)=4 \pi n r_{-2}^{3} \rho_{-2} e^{2 n}(2 n)^{-3 n} \gamma\left(3 n, \frac{r}{r_{-2}}\right) \\
V_{E \text { in }}^{2}(r)=\frac{4 \pi G n r_{-2}^{3} \rho_{-2}}{r} e^{2 n}(2 n)^{-3 n} \gamma\left(3 n, \frac{r}{r_{-2}}\right)
\end{gathered}
$$

where $\gamma(3 n, x)=\int_{0}^{x} e^{-t} t^{3 n-1} d t$, parameter $G$ is the universal gravitational constant, $r_{-2}$ is the radius where the density profile has a slope of $-2, \rho_{-2}$ is 
the local density at that radius and $r$ is the radius from centre of the galaxy. While other dark matter models are described by two free parameters, $r_{s}$ and $\rho_{0}$, a characteristic scale and a characteristic density at that radius, Einasto model involves a third parameter, $n$, the Einasto index which describes the shape of the density profile [44].

\subsubsection{Core-modified profile}

The NFW profile is singular at the galactic center. To avoid the singularity, Brownstein proposed the core-modified profile. The core-modified profile is a profile with constant density in the central core [45]. The density, mass and velocity [46] equations of core-modified profile are as follows:

$$
\begin{gathered}
\rho_{c o m}(r)=\frac{\rho_{0} r_{s}^{3}}{r^{3}+r_{s}^{3}} \\
M_{c o m}(r)=\frac{4}{3} \pi \rho_{0} r_{s}^{3}\left[\ln \left(r^{3}+r_{s}^{3}\right)-\ln \left(r_{s}^{3}\right)\right] \\
V_{c o m}^{2}(r)=\frac{4}{3} \pi G \rho_{0} \frac{r_{s}^{3}}{r}\left[\ln \left(r^{3}+r_{s}^{3}\right)-\ln \left(r_{s}^{3}\right)\right]
\end{gathered}
$$

\subsubsection{DC14 profile}

DC14 profile considers the baryonic feedback on the halo due to the supernovae, and hence modifies the halo profiles [47]. Cintio et al. established the DC14 model, whose profile is defined in terms of the model class $(\alpha, \beta$,

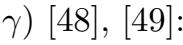

$$
\rho_{\alpha \beta \gamma}(r)=\frac{\rho_{0}}{\left(\frac{r}{r_{s}}\right)^{\gamma}\left[1+\left(\frac{r}{r_{s}}\right)^{\alpha}\right]^{(\beta-\gamma) / \alpha}}
$$

where $\beta$ and $\gamma$ are the inner and outer slopes, respectively, and $\alpha$ describes the transition between the inner and outer regions. These parameters are represented by the equations below:

$$
\begin{aligned}
& \alpha=2.94-\log \left(10^{(X+2.33)-1.08}+10^{(X+2.33) 2.29}\right), \\
& \beta=4.23+1.34 X+0.26 X^{2}, \\
& \gamma=-0.06+\log 10^{(X+2.56)-0.68}+10^{X+2.56}
\end{aligned}
$$

where $\mathrm{X}=\log \left(M_{\text {star }} / M_{\text {halo }}\right)$, is the stellar-to-halo mass (SHM) ratio in logarithm space. The mass [50] and velocity of DC14 profile are as follows: 


$$
\begin{gathered}
M_{D C 14}(r)=4 \pi \rho_{0} r_{s}^{3} \frac{1}{\alpha}(B[a, b+1, \epsilon]+B[a+1, b, \epsilon]) \\
V_{D C 14}^{2}(r)=4 \pi G \rho_{0} \frac{r_{s}^{3}}{r} \frac{1}{\alpha}(B[a, b+1, \epsilon]+B[a+1, b, \epsilon])
\end{gathered}
$$

where $B(a, b, x)=\int_{0}^{x} t^{\alpha-1}(1-t)^{b-1} d t$ is the incomplete Beta function and $a=(3-\gamma) / \alpha, b=(\beta-3) / \alpha$ and $\epsilon=\frac{\left(r / r_{s}\right)^{\alpha}}{\left(1+\left(r / r_{s}\right)^{\alpha}\right)}$. This equation only works for the SHM ratio within $-4.1<\mathrm{X}<-1.3$, due to the fact that this is the range where the supernovae feedback is significant and dominant [47]. At X $<-4.1$, the energy released by supernova is insufficient to modify the initial cuspy profile, while at $\mathrm{X}>-1.3$, the feedback due to active galactic nuclei might start to dominate [50].

\subsubsection{CoreNFW}

A coreNFW halo [51] is essentially a NFW halo which transforms an inner cusp into a finite central core by a spherically symmetric function $f^{n}$ that models the effects of supernova feedback [52]. The mass of coreNFW is defined as:

$$
M_{c N F W}(<r)=M_{N F W}(<r) f^{n}(r)
$$

with

$$
f(r)=\left[\tanh \left(\frac{r}{r_{s}}\right)\right]
$$

The strength of the core is determined by the parameter $n$, which ranges between $0<n \leq 1$. The equation of $\mathrm{n}$ is as follows:

$$
n=\tanh \left(\kappa \frac{t_{S F}}{t_{d y n}}\right)
$$

where $\kappa$ is a tunning parameter and $t_{S F}$ is the star formation time of the galaxy. We set $\kappa=0.04$ and $t_{S F}=14$ Gyrs as suggested by the simulations of Read et al. [51]. The dynamical time, $t_{d y n}$ is the duration of 1 circular orbit at the scale radius in the unmodified NFW halo:

$$
t_{d y n}=2 \pi \sqrt{\frac{r_{s}^{3}}{\left(G M_{N F W}\left(r_{s}\right)\right)}}
$$

Hence, the mass and velocity of coreNFW profile are defined as:

$$
\begin{gathered}
M_{c N F W}(<r)=4 \pi \rho_{0} r_{s}^{3}\left(\ln \left(1+\frac{r}{r_{s}}\right)-\frac{r}{r_{s}+r}\right) f^{n}(r) \\
V_{c N F W}^{2}(<r)=4 \pi G \rho_{0} \frac{r_{s}^{3}}{r}\left(\ln \left(1+\frac{r}{r_{s}}\right)-\frac{r}{r_{s}+r}\right) f^{n}(r)
\end{gathered}
$$




\subsubsection{Lucky13}

Lucky13 is a new semi-empirical profile constructed from Equation (21), the $(\alpha, \beta, \gamma)$ models by Li et al. [50]. They considered the transition parameter $\alpha$ $=1, \gamma=0$ to reach a finite core and $\beta=3$ to get the same decreasing rate as the NFW profile at large radii. The density, mass and velocity of Lucky13 [50] are as follows:

$$
\begin{gathered}
\rho_{130}=\frac{\rho_{0}}{\left[1+\left(\frac{r}{r_{s}}\right)\right]^{3}} \\
M_{130}(r)=4 \pi \rho_{0} r_{s}^{3}\left[\ln \left(1+\frac{r}{r_{s}}\right)+\frac{2}{\left(1+\frac{r}{r_{s}}\right)}-\frac{2}{2\left(1+\frac{r}{r_{s}}\right)^{2}}-\frac{3}{2}\right] \\
V_{130}^{2}(r)=4 \pi G \rho_{0} \frac{r_{s}^{3}}{r}\left[\ln \left(1+\frac{r}{r_{s}}\right)+\frac{2}{\left(1+\frac{r}{r_{s}}\right)}-\frac{2}{2\left(1+\frac{r}{r_{s}}\right)^{2}}-\frac{3}{2}\right]
\end{gathered}
$$

\subsection{Star Velocity}

Most of the luminous mass is occupied by stars, and the rest (i.e. less than 10 $\%$ ) is filled with interstellar gases. Hence, the star disc luminosity distribution roughly represents the luminous mass distribution [53]. To estimate the stellar star luminosity, we use the velocity of star disc derived by Freeman [54,55], which is described by the equation below:

$$
V_{\text {star }}^{2}=\frac{G M_{D} x^{2}}{2 R_{D}}\left(I_{o} K_{o}-I_{1} K_{1}\right)
$$

where $G$ is the universal gravitational constant, $I_{n}$ and $K_{n}$ are the modified Bessel functions of the first and second kinds computed at $x / 2$ and $x=\frac{r}{R_{D}}$, $M_{D}$ is the star mass and $R_{D}$ is the star scale length with value in kpc. The star scale length for NGC 4321 that we adopted is $75 \operatorname{arcsec}$ [56], which is equivalent to $6.22 \mathrm{kpc}$.

The bulge in M100 is small compared to the disks hence no bulge-disk decomposition was adopted [57]. The bulge of M100 does not contribute much to the surface brightness of the disk and the surface brightness have a linear correlation with stellar mass [58]. This indicates that the bulge of M100 does not contribute much to the stellar mass and can be omitted.

\subsection{Gas velocity}

Taking account of the gas component and if its mass is significant, it is possible to use photometry to calculate the rotation curve of the baryonic disc component by matching it with observations to estimate the effect of dark matter 


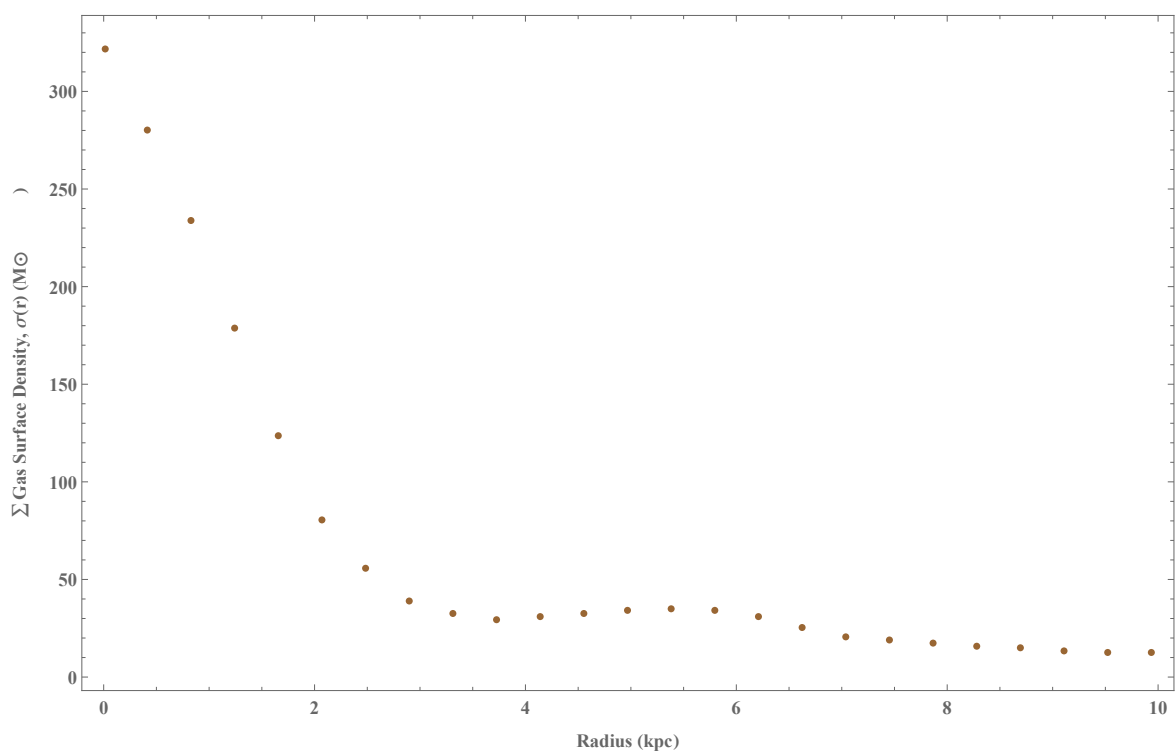

Fig. $6 \sum$ Gas surface density graph

on the disc dynamics [59]. We compute the mass of gas from the equation [60] that is given below:

$$
M_{\text {gas }}(r)=2 \pi \int_{0}^{r} r \sigma(r) d r
$$

where $r$ is the radius from centre of the galaxy and $\sigma(r)$ is the surface density. From Equation (35), we applied the non-linear fitting method to the curve of total gas surface density. The steps of obtaining the gas velocity by using software Mathematica are as below:

i Adopt the surface density profile total gas data from $\mathrm{T}$. Wong and $\mathrm{L}$. Blitz [61] as shown in Figure 6.

ii Plot the best fitting curve that computed from several trial values of the parameter and equation as shown in Figure 7.

iii Calculate the goodness of fit, $\chi^{2}$ to indicate the best-fitting curves and it will show the best model of $\Sigma$ gas surface density, $\sigma(r)$.

iv Apply the best model of $\Sigma$ gas surface density, $\sigma(r)$ to Equation (35) to calculate the mass of gas for each radius.

$\mathrm{v}$ From the mass of gas for each radius, then derive the velocity for each radius by considering the equation

$$
v=\sqrt{\frac{G M(<r)}{r}}
$$

and the result is as shown in Figure 8. 


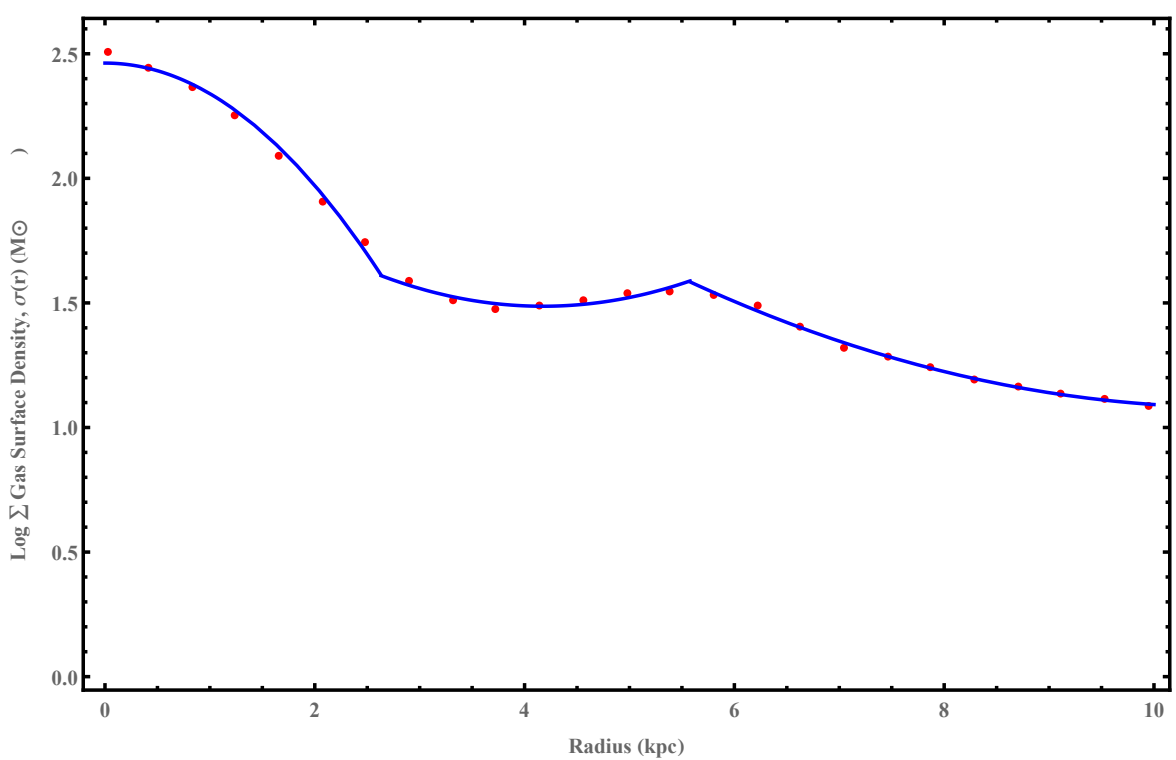

Fig. 7 The best-fitting graph with $\chi^{2}=0.94$. The red dot point represents gas surface density and the blue line represents the best fitting line.

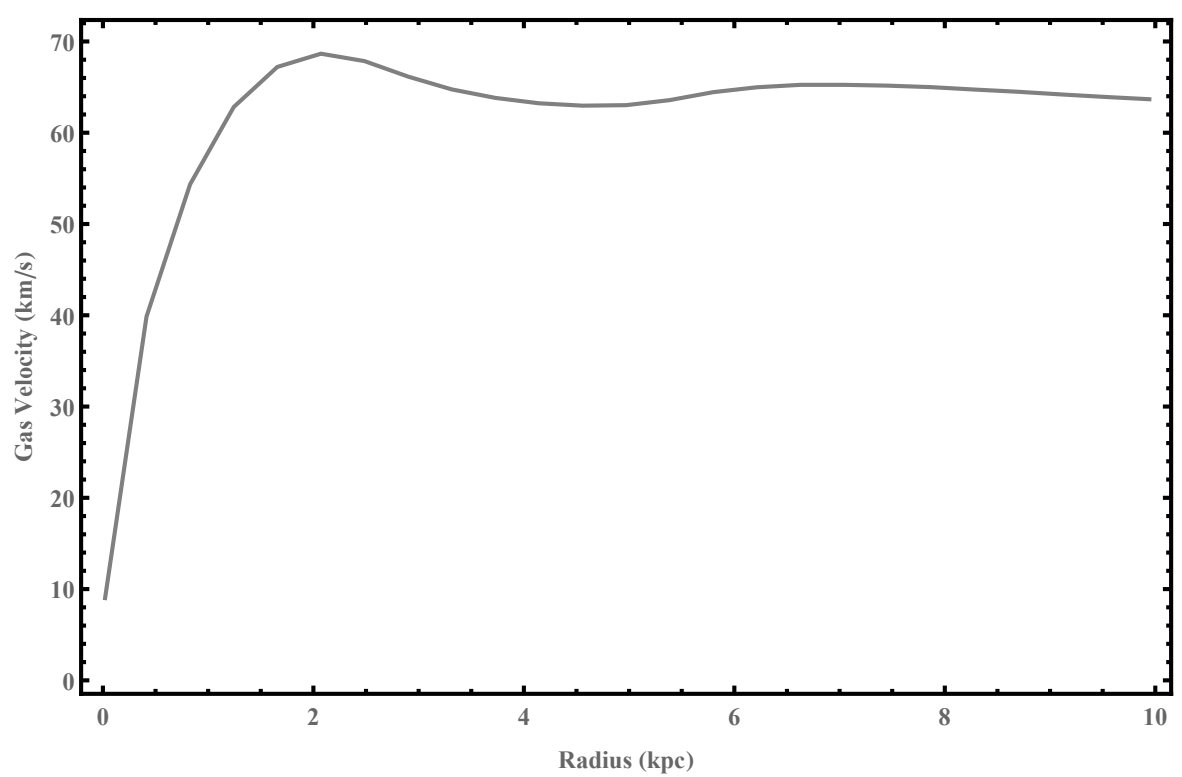

Fig. 8 Gas velocity graph 


\begin{tabular}{|c|c|c|c|}
\hline Dark Matter Profile & $M_{D}\left(M_{\odot}\right)$ & $\begin{array}{l}\rho_{0}\left(\rho_{-2} \text { for Einasto }\right) \\
\left(M_{\odot} k p c^{-3}\right)\end{array}$ & $\begin{array}{c}r_{s}\left(r_{-2} \text { for }\right. \\
\text { Einasto })(\mathrm{kpc})\end{array}$ \\
\hline $\begin{array}{c}\text { Pseudoisothermal } \\
\text { profile }\end{array}$ & $(5.46 \pm 12.97) \times 10^{10}$ & $(1.98 \pm 1.23) \times 10^{8}$ & $2.55 \pm 0.24$ \\
\hline Burkert profile & $(5.56 \pm 4.53) \times 10^{10}$ & $(1.62 \pm 7.93) \times 10^{7}$ & $5.72 \pm 14.61$ \\
\hline NFW profile & $(5.43 \pm 2.82) \times 10^{10}$ & $(1.48 \pm 0.53) \times 10^{6}$ & $66.77 \pm 21.88$ \\
\hline Moore profile & $(5.56 \pm 7.94) \times 10^{10}$ & $(1.20 \pm 10.11) \times 10^{6}$ & $43.88 \pm 251.20$ \\
\hline Einasto profile & $(5.50 \pm 6.21) \times 10^{10}$ & $(3.87 \pm 1.38) \times 10^{7}$ & $8.44 \pm 1.84$ \\
\hline core-modified profile & $(5.26 \pm 6.88) \times 10^{10}$ & $(5.57 \pm 2.00) \times 10^{7}$ & $4.10 \pm 0.25$ \\
\hline DC14 profile & $(2.43 \pm 0.024) \times 10^{11}$ & $(7.47 \pm 0.0061) \times 10^{8}$ & $0.037 \pm 0.0049$ \\
\hline coreNFW profile & $(5.57 \pm 12.89) \times 10^{10}$ & $(6.43 \pm 4.17) \times 10^{7}$ & $5.23 \pm 0.36$ \\
\hline Lucky13 profile & $(2.43 \pm 0.044) \times 10^{11}$ & $(0.42 \pm 5.48) \times 10^{7}$ & $5.08 \pm 32.96$ \\
\hline
\end{tabular}

Table 5: Free parameter obtained for the nine dark matter profiles

\section{Result \& Discussion}

This section is organized as follows: In subsection 3.1 we present the free parameter obtained and nonlinear fitting rotation curve. Then we illustrate the chi-square test and the mass of dark matter in subsection 3.2. The nine dark matter profiles analysis will be discussed one by one in subsections 3.1 and 3.2 .

\subsection{Free parameter and nonlinear fitting rotation curve}

By referring to Equation (1), where $V_{\text {rot }}^{2}=V_{H I}^{2}$ from VLA data, $V_{\text {gas }}^{2}$ derived from total gas surface density data, while for star and dark matter velocity we will use nonlinear fitting rotation curve method to find the most accurate free parameter. For star velocity $V_{\text {star }}^{2}$, we will use Equation (34) while for dark matter velocity $V_{D M}^{2}$, we will use Equations (5), (8), (11), (14), (17), (20), (24), (30), (33) for Pseudoisothermal, Burkert, NFW, Moore, Einasto, core-modified, DC14, coreNFW and Lucky13 profiles respectively.

We calculated the free parameters from the best fit that we have achieved. The free parameters of star mass $M_{D}$, scale density $\rho_{0}$ and scale radius $r_{s}$ are obtained using the Pseudoisothermal, Burkert, NFW, Moore, core-modified, DC14, coreNFW and Lucky13 profiles. Meanwhile the radius where density profile has a slope of $-2, r_{-2}$ and the local density at that radius, $\rho_{-2}$ is obtained using the Einasto profile. The third free parameter of Einasto profile is obtained using Einasto index that is fitted to $\mathrm{n}=0.77 \pm 0.027$. The other free parameters fitting for the nine dark matter profiles are listed in Table 5. Next, by using the free parameter obtained in Table 5 , we plot the rotation curves of the total rotational velocity with gas, star and dark matter halo velocities for the nine dark matter profiles, are given in Figure 9.

By referring to Figure 9, the rotation curve fitting of DC14 and Lucky13 is rejected, due to the unrealistic low of dark matter velocity for the overall $10 \mathrm{kpc}$ radius. The reason behind the unsuitability of DC14 for this galaxy is because the DC14 equation only works for the SHM ratio within $-4.1<\mathrm{X}$ $<-1.3$ [47]. During the fitting process, we used $\mathrm{X}$ as a free parameter and 


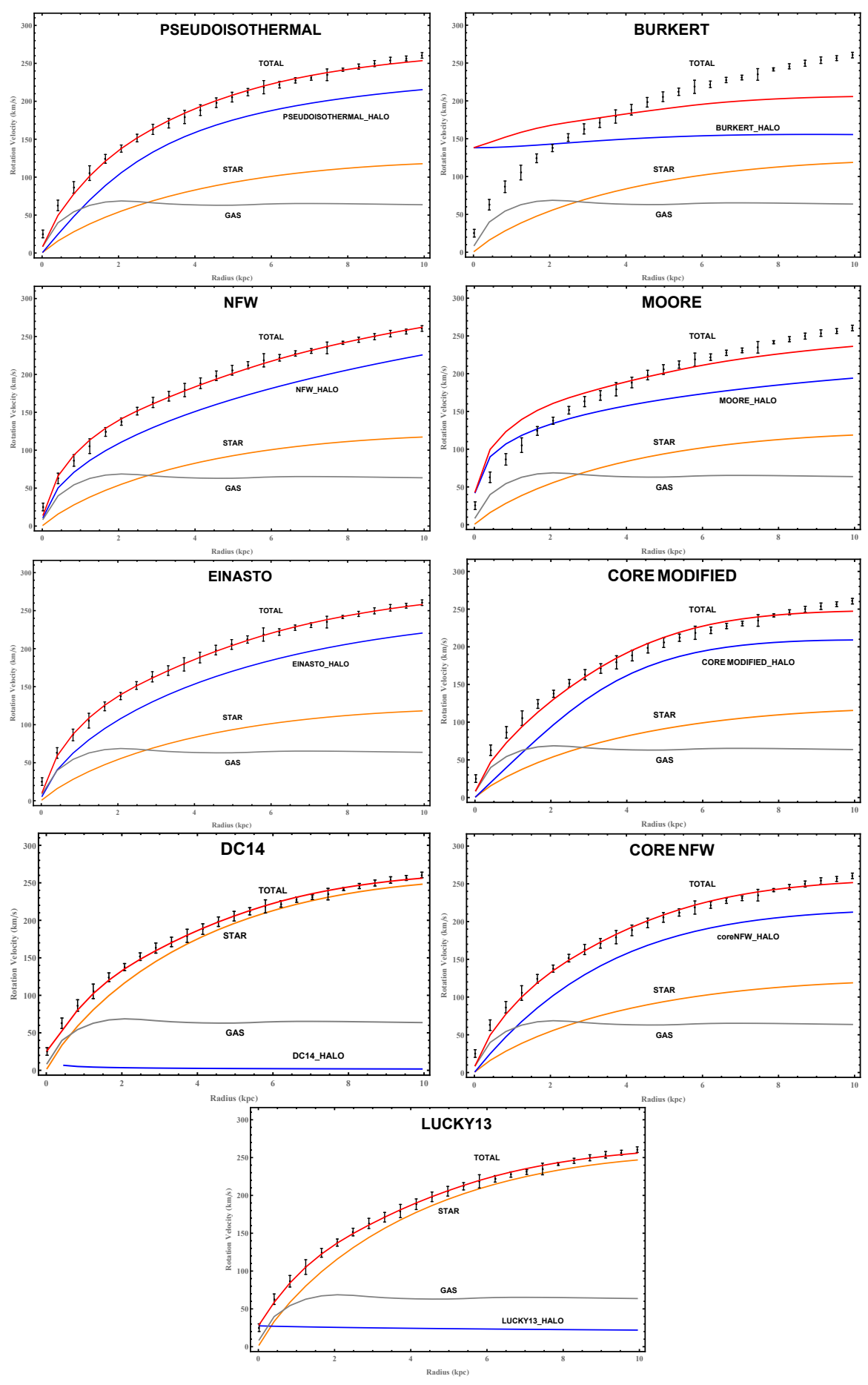

Fig. 9 The nonlinear rotation curve of NGC 4321 by using the nine dark matter profiles. The black and red color represents the total rotation velocity and best fitting line, while the grey, orange and blue color represent gas, star and dark matter halo velocities respectively. 
performed the fitting within the range of -4.1 and -1.3 but none of the values were able to fit the rotation curve well. We then calculated the SHM ratio by applying the relation $\mathrm{X}=\log$ (Mstar/Mhalo) and the masses of the star and dark matter from the five accepted dark matter profiles (Table 7). The calculation for all five accepted profiles showed that the limit of $\mathrm{X}>-1.3$ for this galaxy, which was found to be beyond the limit of the SHM ratio range. Meanwhile, for the Lucky13 profile, this new semi-empirical profile changes the value of alpha, beta and gamma of the $(\alpha, \beta . \gamma)$ model. The gamma set to 0 to reach a finite core, beta set to 3 to get the same decreasing rate as the NFW profile at large radii and transition parameter alpha is set to 1 [50]. These changes within the new semi-empirical profile are not suitable for this galaxy.

According to the previous researches, the Burkert profile is statistically more suitable for the dark matter-dominated dwarf galaxies [34]. Burkert profile revises the density law of Pseudoisothermal profile in the inner region and diverge logarithmically for larger radii. By referring to Figure 9, the Burkert profile reveals the existence of high dark matter velocity $138 \mathrm{kms}^{-1}$ within the inner region close to $0 \mathrm{kpc}$ and increases gradually until the large radii of this galaxy. However, the HI observation data shows the total rotational velocity is only $25 \mathrm{kms}^{-1}$ within the inner region close to $0 \mathrm{kpc}$, hence the mismatch between observation data and Burkert profile suggests that Burkert profile is not suitable for this spiral galaxy.

Moore profile behaves similarly to NFW profile at large radii but it is steeper than NFW profile at smaller radii [39]. By referring to Figure 9, the dark matter velocity of the Moore profile has the steepest rise in the inner region compare to other profiles (except DC14 and Lucky13 profiles). The steepness in the Moore profile leads to its dark matter velocity to be found as higher than the total rotational velocity in the inner region. Even as its steepness reduces after radius $0.5 \mathrm{kpc}$, the dark matter velocity is still higher than the total rotational velocity below the radius $2.0 \mathrm{kpc}$. After radius 2.0 $\mathrm{kpc}$, the dark matter velocity of Moore profile is continuously lower than the total rotational velocity until the large radii of the galaxy. However, our result for the dark matter velocity of Moore profile that is found to be higher than the total rotational velocity before radius $2.0 \mathrm{kpc}$ suggests that Moore profile is not suitable for this galaxy too.

For the remaining five accepted dark matter profiles, it is difficult to determine which dark matter model is better by looking at the figure only. Hence, we will do a chi-square test calculation first before making dark matter model analysis.

\subsection{Chi-square test and the mass of dark matter}

We implemented the chi-square test to test the goodness-of-fit of the rotation curve fitting. The goodness-of-fit parameter, $\chi^{2}$ to be minimized is [62]: 


\begin{tabular}{|l|l|l|}
\hline Dark Matter Profile & Model & $\chi_{\text {red }}^{2}$ \\
\hline \multirow{4}{*}{ Cored } & Pseudoisothermal profile & 1.25 \\
\cline { 2 - 3 } & Einasto profile & 0.52 \\
\cline { 2 - 3 } & core-modified profile & 3.29 \\
\cline { 2 - 3 } & coreNFW profile & 1.64 \\
\hline Cuspy & NFW profile & 0.49 \\
\hline
\end{tabular}

Table 6: $\chi_{\text {red }}^{2}$ for five dark matter profiles

$$
\chi^{2}=\sum_{i=1}^{N} \frac{1}{\sigma_{i}^{2}}\left[y_{i}-y\left(x_{i}, a, b, c\right)\right]^{2}
$$

where $\mathrm{N}$ are observed data points, $\sigma_{i}$ is the uncertainty in $y_{i}, y_{i}$ is the observed rotation curve, $y\left(x_{i}, a, b, c\right)$ are the values of the model function calculated at $x_{i}, x_{i}$ is the radius from the galactic centre and $\mathrm{a}, \mathrm{b}, \mathrm{c}$ are the fit parameters.

Then we performed the goodness-of-fit test by implementing the reduced chi-square:

$$
\chi_{\text {red }}^{2}=\frac{\chi^{2}}{v}
$$

where $v=N-N_{c}, \mathrm{~N}$ is the number of data points and $N_{c}$ is the number of fit parameters. In principle, a value of $\chi_{r e d}^{2}=1$ indicates that the extent of the match between the observations data and the value of the estimates is in accord with the error variance [62].

All reduced chi-square, $\chi_{\text {red }}^{2}$ for the five accepted dark matter profiles are shown in Table 6. Generally, we achieved $0.40<\chi_{\text {red }}^{2}<1.70$ for four out of five dark matter profiles. We found one profile that achieved the $\chi_{\text {red }}^{2}$ closest to 1 , which is Pseudoisothermal profile. For cored profiles, the core-modified profile achieved the highest $\chi_{\text {red }}^{2}$ with 3.29 and followed by coreNFW profiles with $\chi_{\text {red }}^{2}$ of 1.64. While the Einasto and cuspy NFW profile achieved similar lowest $\chi_{\text {red }}^{2}$ with 0.52 and 0.49 , respectively.

Next, we will continue our five accepted dark matter model analysis by referring to the results from the rotation curve in Figure 9 rotation curve and the reduced chi-square test in Table 6 .

Core-modified is a profile with constant density in the central core to avoid singularity in the galactic center [45]. However, the constant density in the central core presented very little flexibility for this profile during the fitting process in the rotation curve modeling. During rotation curve fitting, we found that this profile has certain limitations where less fitting changes that can be made especially in the central core of the galaxy. For the large radii of the galaxy, the increasing rate of dark matter velocity is declining and becomes almost constant after $7.5 \mathrm{kpc}$ radius. The dark matter velocity is normally supposed to continue increasing as the total rotational velocity increases throughout. The increment of dark matter velocity in the large radii of the galaxy can be seen in NFW, coreNFW, Einasto and Pseudoisothermal profiles. This dark matter profile is found to be able to fit the galaxy and the calculated dark 
matter velocity is reasonably better when compare to the other four rejected dark matter profiles. However, the limited flexibility in the core and the constant dark matter velocity in the large radii make the core-modified profile is worse than other four accepted dark matter profiles and only achieved a $\chi_{\text {red }}^{2}$ of 3.29 .

A coreNFW halo is essentially a NFW halo which transforms an inner cusp into a finite central core [51]. The change of inner cusp into a finite central core of this profile have brought improvement in the rotation curve fitting in the inner region of this galaxy. By referring to the inner region of this profile, the increasing dark matter velocity is less steep than the cuspy NFW profile. The steepness of dark matter velocity has made this profile to have good fit with total rotational velocity in the inner region of the galaxy. However, the increasing rate of dark matter velocity is found to be declining beyond $8 \mathrm{kpc}$ radius, causing unsuitability of fitting with the continuously increasing total rotational velocity. This issue causes the coreNFW profile to be considered as not as good as Peudoisothermal profile and achieved a $\chi_{\text {red }}^{2}$ of 1.64 .

The NFW profile is called 'universal' because it works for a large variety of halo masses, same shape of initial density fluctuation spectrum, from individual galaxies to the halos of galaxy clusters [63] and this leads to the NFW profile that fits well to this galaxy as well. However, the NFW profile has the cuspy halo problem that increases steeply at small radii [64]. This problem can be seen in Figure 9, the inner region of the NFW profile is steeper than the coreNFW, Pseudoisothermal and Einasto profiles. From the inner region $0 \mathrm{kpc}$ to $1 \mathrm{kpc}$, the steepness of the NFW profile causes the rotational velocity of halo, star and gas to mismatch with the total rotational velocity. This mismatch causes the fitting of NFW profile to be achieved $\chi_{\text {red }}^{2}$ of 0.49 , which is slightly not as good as the case for the Einasto profile.

High-resolution N-body CDM simulations indicate that nonsingular threeparameter models such as the Einasto profile perform better than the singular two-parameter models such as Pseudoisothermal and NFW profiles, providing an excellent fit to a wide range of dark matter haloes [65]. The Einasto profile involves a third parameter, n, the Einasto index, which describes the shape of the overall profile distribution, larger values of $n$ results in steeper inner profiles and shallower outer profiles [66]. In order to have the best-fitting with the total rotational velocity of this galaxy, the inner profile needs to be shallower and the outer profile needs to be steeper. In our case for this paper, the third parameter, $\mathrm{n}$, acts a very important role to fulfill the criteria of the fitting, where we are able to use smaller values of $n$ to make the inner profile shallower and the outer profile steeper (Figure 9). Furthermore, these three parameters model allows the profile to be tailored to each individual halo, thereby yielding improved fits [67]. This can be seen in Figure 9 where each individual halo is well fitted with total rotational velocity. However, the addition of the third parameter and the profile tailored to each individual halo causes the Einasto profile to ultimately overfits the data with the value $\chi_{\text {red }}^{2}$ of 0.52 .

Pseudoisothermal profile is a commonly used model for dark matter halo analysis and is often seen to better fit the galactic rotation curve than the 


\begin{tabular}{|l|l|l|l|}
\hline $\begin{array}{l}\text { Dark Matter } \\
\text { Profile }\end{array}$ & Model & Mass of star $M \odot)$ & Mass of dark matter $(M \odot)$ \\
\hline \multirow{4}{*}{ Cored } & Pseudoisothermal & $(3.23 \pm 7.66) \times 10^{10}$ & $(1.08 \pm 0.0083) \times 10^{11}$ \\
\cline { 2 - 4 } & Einasto & $(3.25 \pm 3.67) \times 10^{10}$ & $(1.13 \pm 0.00012) \times 10^{11}$ \\
\cline { 2 - 4 } & core-modified & $(3.11 \pm 4.07) \times 10^{10}$ & $(1.01 \pm 0.00035) \times 10^{11}$ \\
\cline { 2 - 4 } & coreNFW & $(3.29 \pm 7.61) \times 10^{10}$ & $(1.05 \pm 0.0014) \times 10^{11}$ \\
\hline Cuspy & NFW & $(3.21 \pm 1.66) \times 10^{10}$ & $(1.19 \pm 0.10) \times 10^{11}$ \\
\hline
\end{tabular}

Table 7: Total dark matter mass within radius $10 \mathrm{kpc}$ for the dark matter profiles

NFW model $[68,69]$ and this works for this spiral galaxy as well. By looking at Figure 9, the overall Pseudoisothermal profile distribution fits very well and matches with Equation (1). In addition, the Pseudoisothermal rotation curve has a linear growth at the inner region then becomes flat at large radii [70]. This trend can be seen on this galaxy too, where the rotational velocity of the Pseudoisothermal profile in the inner region below $1 \mathrm{kpc}$ increases linearly and the rotational velocity becomes flat at large radii. The linearity at the inner region and the flatness at the large radii of the fitting characteristic are very suitable for this galaxy. This causes the Pseudoisothermal to achieve the best fitting among the nine dark matter profiles with $\chi_{\text {red }}^{2}$ of 1.25 , which is the nearest to 1 .

Based on the free parameters obtained as shown in Table 5, we calculated the total star mass and dark matter mass within radius $10 \mathrm{kpc}$ for each dark matter profile as shown in Table 7. We obtained the star velocity from Equation (34) and subsequently calculated the star mass with Equation (36), while the mass of dark matter is calculated by applying the Equations (4), (10), (16), (19), (29) for Pseudoisothermal, NFW, Einasto, core-modified and coreNFW profiles, respectively.

The total dark matter mass for the five accepted dark matter profiles within radius $10 \mathrm{kpc}$ in this galaxy is in the range from $(1.01 \pm 0.00035) \times 10^{11} \mathrm{M} \odot$ to $(1.19 \pm 0.10) \times 10^{11} M \odot$. By taking consideration of the $\chi_{\text {red }}^{2}$, the Pseudoisothermal, Einasto, coreNFW and NFW profiles achieved the $\chi_{\text {red }}^{2}$ within the range of $0.40<\chi_{\text {red }}^{2}<1.70$. The mass of dark matter in this galaxy within the range of these four profiles is calculated to be from $(1.05 \pm 0.0014) \times 10^{11} \mathrm{M} \odot$ to $(1.19 \pm 0.10) \times 10^{11} M \odot$.

\section{Conclusion}

For this galaxy, we analyzed the dark matter of the galaxy NGC 4321 by using the nine dark matter profiles. The nine dark matter profiles consist of seven core and two cuspy profiles. The core profiles are namely Pseudoisothermal, Burkert, Einasto, core-modified, DC14, coreNFW and Lucky13 profiles. The cuspy profiles are NFW and Moore profiles. We analyzed in detail how each dark matter profile performs in this galaxy. We rejected four dark matter profiles while five dark matter profiles are accepted for this galaxy. DC14 profile is rejected due to the stellar-to-halo mass ratio that is found to be out of the range of the profile condition. Lucky13 profile is rejected due to the 
unsuitability of the galaxy model setting of this new semi-empirical profile. The revised density law of Burkert profile causes the dark matter velocity to mismatch with regards to the total rotational velocity starting from the inner region of the galaxy. Further analysis showed that the Moore profile has the steepest rise in the inner region among the five dark matter profiles and this causes the dark matter velocity in the inner region to be higher than the total rotational velocity below the radius $2.0 \mathrm{kpc}$. These reasons suggest that these four dark matter profiles are not suitable for this galaxy.

For the remaining five accepted dark matter profiles, we analyzed the dark matter velocity together with the reduced chi-square test. The constant core of the core-modified profile causes the limited flexibility on fitting and hence producing a large value of $\chi_{r e d}^{2}$. The NFW profile cuspy halo has a weakness, where the inner region increases steeply at small radii, resulting in the total rotational velocity of halo, star and gas to be higher than the total rotational velocity by $\mathrm{HI}$ observed data at the inner region of this galaxy. The change of inner cusp into a finite central core of the coreNFW profile improved the NFW profile to become less steep in the inner region of the galaxy and this resulted in a better rotation curve fitting. The additional third parameter of the Einasto profile brings the flexibility of adjustment in the inner and outer radii of the galaxy and this enabled the Einasto profile to generate a better overall fitting. The Pseudoisothermal profile produced linear rotational velocity in the inner region and flat rotational velocity at the large radii of this galaxy. This fitting characteristic suits this galaxy very well by producing the best fitting among the nine dark matter profiles with a $\chi_{\text {red }}^{2}$ of 1.25 .

Generally, the NFW, coreNFW, Einasto and Pseudoisothermal profiles achieve relatively good fittings and the mass of dark matter for these four profiles is calculated to be within the range from $(1.05 \pm 0.0014) \times 10^{11} M \odot$ to $(1.19 \pm 0.10) \times 10^{11} M \odot$. Ultimately, our results showed that the Pseudoisothermal profile achieved the best fitting among the nine dark matter profiles and the dark matter mass of this galaxy obtained through the use of this profile is calculated as $(1.08 \pm 0.0083) \times 10^{11} M \odot$.

Acknowledgements The authors would like to acknowledge the funding by the University of Malaya (FG033-017AFR). 


\section{References}

1. Albert Bosma. 21-cm line studies of spiral galaxies. i-observations of the galaxies ngc 5033, 3198, 5055, 2841, and 7331. The Astronomical Journal, 86:1791-1824, 1981.

2. Massimo Persic, Paolo Salucci, and Fulvio Stel. The universal rotation curve of spiral galaxies-i. the dark matter connection. Monthly Notices of the Royal Astronomical Society, 281(1):27-47, 1996.

3. Vera C Rubin, W Kent Ford Jr, and Norbert Thonnard. Rotational properties of 21 sc galaxies with a large range of luminosities and radii, from ngc $4605 / \mathrm{r}=4 \mathrm{kpc} /$ to ugc 2885/r= $122 \mathrm{kpc}$. The Astrophysical Journal, 238:471-487, 1980.

4. Fiorenza Donato, Gianfranco Gentile, Paolo Salucci, Christiane Frigerio Martins, MI Wilkinson, Gerard Gilmore, EK Grebel, Andreas Koch, and Rosemary Wyse. A constant dark matter halo surface density in galaxies. Monthly Notices of the Royal Astronomical Society, 397(3):1169-1176, 2009.

5. Vera C Rubin and W Kent Ford Jr. Rotation of the andromeda nebula from a spectroscopic survey of emission regions. The Astrophysical Journal, 159:379, 1970.

6. Debra Meloy Elmegreen and Bruce G Elmegreen. Arm classifications for spiral galaxies. The Astrophysical Journal, 314:3-9, 1987.

7. JH Knapen, I Shlosman, CH Heller, RJ Rand, JE Beckman, and M Rozas. Kinematics of ionized and molecular hydrogen in the core of m100. The Astrophysical Journal, 528(1):219, 2000.

8. J Cepa, JE Beckman, JH Knapen, N Nakai, and N Kuno. Star formation in the spiral arms of ngc 4321. i-co observations. The Astronomical Journal, 103:429-435, 1992

9. Yoshiaki Sofue. Rotation and mass in the milky way and spiral galaxies. Publications of the Astronomical Society of Japan, 69(1), 2017.

10. WJG De Blok, Fabian Walter, Elias Brinks, C Trachternach, SH Oh, and RC Kennicutt Jr. High-resolution rotation curves and galaxy mass models from things. The Astronomical Journal, 136(6):2648, 2008.

11. S Garcia-Burillo, MJ Sempere, and F Combes. Gas response at the resonances in the grand design spiral ngc 4321. Astronomy and Astrophysics, 287:419, 1994.

12. JH Knapen, JE Beckman, CH Heller, I Shlosman, and RS De Jong. The central region in m100: Observations and modeling. arXiv preprint astro-ph/9506098, 1995.

13. Johan H Knapen. Large-scale star formation patterns in spiral arms. Publications of the Astronomical Society of the Pacific, 105(685):323, 1993.

14. Johan H Knapen, Jordi Cepa, John E Beckman, M Soledad del Rio, and Alan Pedlar. Star formation in the spiral arms of ngc 4321. ii. h i distribution and kinematics. The Astrophysical Journal, 416:563, 1993.

15. Aeree Chung, JH Van Gorkom, Jeffrey DP Kenney, Hugh Crowl, and Bernd Vollmer. Vla imaging of virgo spirals in atomic gas (viva). i. the atlas and the hi properties. The Astronomical Journal, 138(6):1741, 2009.

16. Yoshiaki Sofue, Y Tutui, M Honma, A Tomita, T Takamiya, J Koda, and Y Takeda. Central rotation curves of spiral galaxies. The Astrophysical Journal, 523(1):136, 1999.

17. Olivier Daigle, C Carignan, P Amram, O Hernandez, L Chemin, C Balkowski, and $\mathrm{R}$ Kennicutt. $\mathrm{H} \alpha$ kinematics of the sings nearby galaxies survey-i. Monthly Notices of the Royal Astronomical Society, 367(2):469-512, 2006.

18. A Castillo-Morales, Jorge Jiménez-Vicente, E Mediavilla, and E Battaner. Non-circular motion evidence in the circumnuclear region of m100 (ngc 4321). Monthly Notices of the Royal Astronomical Society, 380(2):489-498, 2007.

19. Israa Abdulqasim Mohammed Ali, Chorng-Yuan Hwang, and Zamri Zainal Abidin. Dark matter in the central region of the spiral galaxy ngc 4321 . arXiv preprint arXiv:1812.05918, 2018.

20. Noemi Frusciante, Paolo Salucci, Daniele Vernieri, John M Cannon, and Ed C Elson. The distribution of mass in the orion dwarf galaxy. Monthly Notices of the Royal Astronomical Society, 426(1):751-757, 2012.

21. Xiao-Sheng Xin and Xing-Wu Zheng. A revised rotation curve of the milky way with maser astrometry. Research in Astronomy and Astrophysics, 13(7):849, 2013.

22. Miguel Pato and Fabio Iocco. The dark matter profile of the milky way: a non-parametric reconstruction. The Astrophysical Journal Letters, 803(1):L3, 2015. 
23. Toky H Randriamampandry and Claude Carignan. Galaxy mass models: Mond versus dark matter haloes. Monthly Notices of the Royal Astronomical Society, 439(2):21322145, 2014.

24. Chi Yuan and Chao-Lin Kuo. Spiral structure in the central disks of ngc 1068 and m100. The Astrophysical Journal, 497(2):689, 1998.

25. Jazeel H Azeez, C-Y Hwang, Zamri Z Abidin, and Zainol A Ibrahim. Kennicutt-schmidt law in the central region of ngc 4321 as seen by alma. Scientific reports, 6:26896, 2016

26. Shouta Oikawa and Yoshiaki Sofue. Rotation curve anomaly and galactic warp in $\mathrm{m}$ 51. Publications of the Astronomical Society of Japan, 66(4), 2014.

27. DH Rogstad, IA Lockhart, and MCH Wright. Aperture-synthesis observations of hi in the galaxy m83. The Astrophysical Journal, 193:309-319, 1974.

28. KG Begeman. Hi rotation curves of spiral galaxies. i-ngc 3198. Astronomy and Astrophysics, 223:47-60, 1989.

29. Gyula IG Jozsa, Franz Kenn, Uli Klein, and Tom A Oosterloo. Kinematic modelling of disk galaxies-i. a new method to fit tilted rings to data cubes. Astronomy \& Astrophysics, 468(2):731-774, 2007.

30. A Vafaei Sadr, Etienne E Vos, Bruce A Bassett, Zafiirah Hosenie, N Oozeer, and Michelle Lochner. Deepsource: point source detection using deep learning. Monthly Notices of the Royal Astronomical Society, 484(2):2793, 2019.

31. Daniel Shenon Briggs. New Mexico Institute of Mining Technology. PhD thesis, New Mexico Institute of Mining and Technology, 1995.

32. Hugo Martel and Paul R Shapiro. Gravitational lensing by the truncated isothermal sphere model for cosmological halos. i. individual lens properties. arXiv preprint astroph/0207631, 2002.

33. Raul Jimenez, Licia Verde, and S Peng Oh. Dark halo properties from rotation curves. Monthly Notices of the Royal Astronomical Society, 339(1):243-259, 2003.

34. Andreas Burkert. The structure of dark matter halos in dwarf galaxies. The Astrophysical Journal Letters, 447(1):L25, 1995.

35. Paolo Salucci and Andreas Burkert. Dark matter scaling relations. The Astrophysical Journal Letters, 537(1):L9, 2000.

36. N Hashim, ZZ Abidin, UFSU Ibrahim, MSR Hassan, ZS Hamidi, Roslan Umar, and ZA Ibrahim. The nonlinear least square fitting for rotation curve of orion dwarf spiral. Sains Malaysiana, 44(3):457-462, 2015.

37. Julio F Navarro. The structure of cold dark matter halos. In Symposium-international astronomical union, volume 171, pages 255-258. Cambridge University Press, 1996.

38. Dan Coe. Dark matter halo mass profiles. arXiv preprint arXiv:1005.0411, 2010.

39. Jürg Diemand, Ben Moore, and Joachim Stadel. Convergence and scatter of cluster density profiles. Monthly Notices of the Royal Astronomical Society, 353(2):624-632, 2004.

40. Ben Moore, Tom Quinn, Fabio Governato, Joachim Stadel, and George Lake. Cold collapse and the core catastrophe. Monthly Notices of the Royal Astronomical Society, 310(4):1147-1152, 1999.

41. Anatoly Klypin, Andrey V Kravtsov, James S Bullock, and Joel R Primack. Resolving the structure of cold dark matter halos. The Astrophysical Journal, 554(2):903, 2001.

42. Mauro Sereno, Cosimo Fedeli, and Lauro Moscardini. Comparison of weak lensing by nfw and einasto halos and systematic errors. Journal of Cosmology and Astroparticle Physics, 2016(01):042, 2016.

43. Julio F Navarro, Aaron Ludlow, Volker Springel, Jie Wang, Mark Vogelsberger, Simon DM White, Adrian Jenkins, Carlos S Frenk, and Amina Helmi. The diversity and similarity of simulated cold dark matter haloes. Monthly Notices of the Royal Astronomical Society, 402(1):21-34, 2010.

44. Laurent Chemin, WJG De Blok, and Gary A Mamon. Improved modeling of the mass distribution of disk galaxies by the einasto halo model. The Astronomical Journal, 142(4):109, 2011

45. Joel Richard Brownstein. Modified gravity and the phantom of dark matter. arXiv preprint arXiv:0908.0040, 2009.

46. Xin Li, Li Tang, and Hai-Nan Lin. Comparing dark matter models, modified newtonian dynamics and modified gravity in accounting for galaxy rotation curves. Chinese Physics C, 41(5):055101, 2017. 
47. Arianna Di Cintio, Chris B Brook, Aaron A Dutton, Andrea V Macciò, Greg S Stinson, and Alexander Knebe. A mass-dependent density profile for dark matter haloes including the influence of galaxy formation. Monthly Notices of the Royal Astronomical Society, 441(4):2986-2995, 2014.

48. Lars Hernquist. An analytical model for spherical galaxies and bulges. The Astrophysical Journal, 356:359-364, 1990.

49. HongSheng Zhao. Analytical models for galactic nuclei. Monthly Notices of the Royal Astronomical Society, 278(2):488-496, 1996.

50. Pengfei Li, Federico Lelli, Stacy McGaugh, and James Schombert. A comprehensive catalog of dark matter halo models for sparc galaxies. The Astrophysical Journal Supplement Series, 247(1):31, 2020.

51. JI Read, O Agertz, and MLM Collins. Dark matter cores all the way down. Monthly Notices of the Royal Astronomical Society, 459(3):2573, 2016.

52. Flor Allaert, Gianfranco Gentile, and Maarten Baes. Testing baryon-induced core formation in $\lambda$ cdm: A comparison of the dc14 and corenfw dark matter halo models on galaxy rotation curves. Astronomy \& Astrophysics, 605:A55, 2017.

53. Yoshiaki Sofue. Mass distribution and rotation curve in the galaxy. Planets, Stars and Stellar Systems: Volume 5: Galactic Structure and Stellar Populations, pages 985-1037, 2013.

54. Kenneth C Freeman. On the disks of spiral and s0 galaxies. The Astrophysical Journal, 160:811, 1970.

55. Chiara Di Paolo, Paolo Salucci, and Adnan Erkurt. The universal rotation curve of low surface brightness galaxies-iv. the interrelation between dark and luminous matter. Monthly Notices of the Royal Astronomical Society, 490(4):5451-5477, 2019.

56. Rebecca A Koopmann, Jeffrey DP Kenney, and Judith Young. An atlas of h $\alpha$ and $\mathrm{r}$ images and radial profiles of 63 bright virgo cluster spiral galaxies. The Astrophysical Journal Supplement Series, 135(2):125, 2001.

57. JE Beckman, RF Peletier, JH Knapen, RLM Corradi, and LJ Gentet. Scale lengths in disk surface brightness as probes of dust extinction in three spiral galaxies: M51, ngc 3631, and ngc 4321. The Astrophysical Journal, 467:175, 1996.

58. Takashi Ichikawa, Masaru Kajisawa, Toru Yamada, Masayuki Akiyama, Tomohiro Yoshikawa, Masato Onodera, and Masahiro Konishi. Moircs deep survey. v. a universal relation for stellar mass and surface brightness of galaxies. The Astrophysical Journal, 709(2):741, 2010.

59. Anatolii Vladimirovich Zasov, Ann S Saburova, Alexander Valentinovich Khoperskov, and Sergey A Khoperskov. Dark matter in galaxies. Physics-Uspekhi, 60(1):3, 2017.

60. Norsiah Hashim, Mariafelicia De Laurentis, Zamri Zainal Abidin, and Paolo Salucci. Rotation curve with mond and dark matter halo profile for eso138-g014. arXiv preprint arXiv:140\%.0379, 2014.

61. Tony Wong and Leo Blitz. The relationship between gas content and star formation in molecule-rich spiral galaxies. The Astrophysical Journal, 569(1):157, 2002.

62. Philip R Bevington and D Keith Robinson. Data reduction and error analysis for the physical sciences mcgraw-hill. New York, 19692:235-242, 1969.

63. Julio F Navarro, Carlos S Frenk, and Simon DM White. A universal density profile from hierarchical clustering. The Astrophysical Journal, 490(2):493, 1997.

64. WJG De Blok. The core-cusp problem. Advances in Astronomy, 2010, 2010.

65. E Retana-Montenegro, Emmanuel Van Hese, Gianfranco Gentile, Maarten Baes, and F Frutos-Alfaro. Analytical properties of einasto dark matter haloes. Astronomy $\&$ Astrophysics, 540:A70, 2012.

66. Alister W Graham, David Merritt, Ben Moore, Jürg Diemand, and Balša Terzić. Empirical models for dark matter halos. ii. inner profile slopes, dynamical profiles, and $\rho / \sigma 3$. The Astronomical Journal, 132(6):2701, 2006.

67. Julio F Navarro, Eric Hayashi, Chris Power, AR Jenkins, Carlos S Frenk, Simon DM White, Volker Springel, Joachim Stadel, and Thomas R Quinn. The inner structure of $\lambda \mathrm{cdm}$ haloes-iii. universality and asymptotic slopes. Monthly Notices of the Royal Astronomical Society, 349(3):1039-1051, 2004.

68. WJG De Blok, Stacy S McGaugh, and Vera C Rubin. High-resolution rotation curves of low surface brightness galaxies. ii. mass models. The Astronomical Journal, 122(5):2396, 2001. 
69. Rachel Kuzio De Naray, Stacy S McGaugh, WJG De Blok, and A Bosma. Highresolution optical velocity fields of 11 low surface brightness galaxies. The Astrophysical Journal Supplement Series, 165(2):461, 2006.

70. Rachel Kuzio De Naray, Stacy S McGaugh, and WJG De Blok. Mass models for low surface brightness galaxies with high-resolution optical velocity fields. The Astrophysical Journal, 676(2):920, 2008 . 